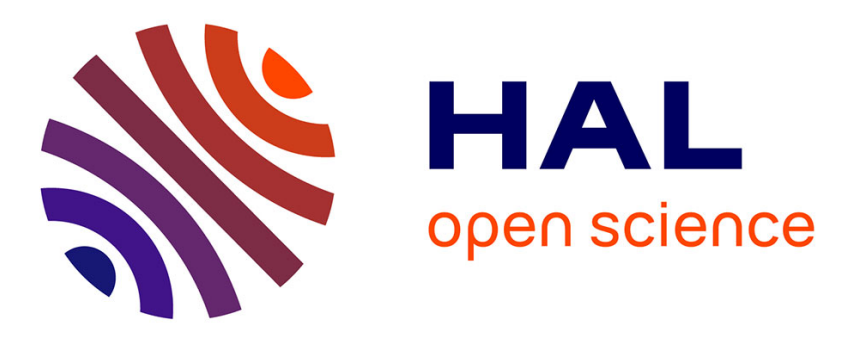

\title{
Wetting of Heterogeneous Surfaces at the Mesoscopic Scale
}

Joël de Coninck, Christophe Dobrovolny, Salvador Miracle-Solé, Jean Ruiz

\section{To cite this version:}

Joël de Coninck, Christophe Dobrovolny, Salvador Miracle-Solé, Jean Ruiz. Wetting of Heterogeneous

Surfaces at the Mesoscopic Scale. Journal of Statistical Physics, 2004, 114, pp.574-604. hal-00138010

\section{HAL Id: hal-00138010 \\ https://hal.science/hal-00138010}

Submitted on 23 Mar 2007

HAL is a multi-disciplinary open access archive for the deposit and dissemination of scientific research documents, whether they are published or not. The documents may come from teaching and research institutions in France or abroad, or from public or private research centers.
L'archive ouverte pluridisciplinaire HAL, est destinée au dépôt et à la diffusion de documents scientifiques de niveau recherche, publiés ou non, émanant des établissements d'enseignement et de recherche français ou étrangers, des laboratoires publics ou privés. 


\title{
Wetting of Heterogeneous Surfaces at the Mesoscopic Scale
}

\author{
Joël De Coninck ${ }^{1}$, Christophe Dobrovolny ${ }^{2}$, \\ Salvador Miracle-Solé ${ }^{3}$, and Jean Ruiz ${ }^{4}$
}

\begin{abstract}
We consider the problem of wetting on a heterogeneous wall with mesoscopic defects: i.e. defects of order $L^{\varepsilon}, 0<\varepsilon<1$, where $L$ is some typical length-scale of the system. In this framework, we extend several former rigorous results which were shown for walls with microscopic defects $[10,11]$. Namely, using statistical techniques applied to a suitably defined semi-infinite Ising-model, we derive a generalization of Young's law for rough and heterogeneous surfaces, which is known as the generalized Cassie-Wenzel's equation. In the homogeneous case, we also show that for a particular geometry of the wall, the model can exhibit a surface phase transition between two regimes which are either governed by Wenzel's or by Cassie's law.
\end{abstract}

KEY WORDS: Wetting, Wenzel's law, Cassie's law, roughness, interfaces.

Preprint CPT-2003/P.4532 revised

${ }^{1}$ Centre de Recherche en Modélisation Moléculaire, Université de Mons-Hainaut, 20 place du Parc, B-7000 Mons, Belgium.

E-mail address: Joel.De.Coninck@galileo.umh.ac.be

${ }^{2}$ Centre de Recherche en Modélisation Moléculaire, Université de Mons-Hainaut, 20 place du Parc, B-7000 Mons, Belgium. Permanent address: Centre de Physique Théorique, CNRS, Luminy case 907, F-13288 Marseille Cedex 9, France.

E-mail address: dobrovol@cpt.univ-mrs.fr

${ }^{3}$ Centre de Physique Théorique, CNRS, Luminy case 907, F-13288 Marseille Cedex 9, France. E-mail address: Salvador.Miracle-Sole@cpt.univ-mrs.fr

${ }^{4}$ Centre de Physique Théorique, CNRS, Luminy case 907, F-13288 Marseille Cedex 9, France. E-mail address: Jean.Ruiz@cpt.univ-mrs.fr 


\section{Introduction}

Surface phenomena play an important role in many fundamental processes and, among them, the wetting of surfaces is a subject of primary importance.

Consider a drop of liquid $B$ in coexistence with a gas phase $A$ on top of the surface $W$. The shape of this drop with a fixed volume of liquid is obtained by minimizing the free energies associated to the three interfaces under consideration. The solution of the corresponding variational problem is given by the Winterbottom's construction.

As a consequence, the contact angle of the droplet with the wall satisfies in the isotropic case the well known Young's equation :

$$
\tau_{A B} \cos \theta=\tau_{A W}-\tau_{B W} \equiv \Delta \tau
$$

where $\tau_{i j},\{i, j\} \in\{A, B, W\}$ is the surface tension between the media $i$ and $j$. In the case of an orientation dependent surface tension for the $A B$-interface, the L.H.S. of the above equations have to be modified: e.g. in dimension $d=2$, one should replace it, by $\cos \theta \tau_{A B}-\sin \theta \frac{d}{d \theta} \tau_{A B}$ (see [8]).

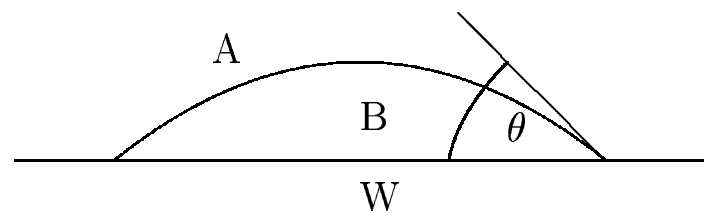

Figure 1: Young's contact angle

The validity of Winterbottom's construction and Young's equations in the frame of Statistical Mechanics has been established in several works: see $[8,9]$ for SOS-models and $[1,24]$ for Ising-like models. The substrate $W$ is usually considered as perfectly flat and homogeneous surface.

When the surface is homogeneous but rough, one usually introduce the roughness as the ratio of the area $A$ of the surface and the area $\bar{A}$ of its projection on the horizontal plane: $r=A / \bar{A}$. In this case the differential wall tension $\Delta \tau$ has to be computed according to the Wenzel's law [29]:

$$
\Delta \tau=r(\Delta \tau)^{\mathrm{flat}}
$$


where $(\Delta \tau)^{\mathrm{flat}}=\tau_{A W}^{\mathrm{flat}}-\tau_{B W}^{\mathrm{flat}}$ is the differential wall tension of the corresponding flat wall.

When the substrate is flat but made of two species $W_{1}$ and $W_{2}$ with concentrations $c_{1}$ and $c_{2}=1-c_{1}$, respectively, we will have:

$$
\Delta \tau=c_{1}(\Delta \tau)_{1}^{\text {flat }}+c_{2}(\Delta \tau)_{2}^{\text {flat }}
$$

where $(\Delta \tau)_{i}^{\mathrm{flat}}=\tau_{A W_{i}}^{\mathrm{flat}}-\tau_{B W_{i}}^{\mathrm{flat}}$. This relation is known as the Cassie's law [7].

When the substrate is both rough and heterogeneous the generalized Cassie-Wenzel's law states:

$$
\left.\Delta \tau=r_{1} c_{1}(\Delta \tau)_{1}^{\mathrm{flat}}+r_{2} c_{2}\right)(\Delta \tau)_{2}^{\mathrm{flat}}
$$

where $r_{i} c_{i}$ is the ratio of the non planar surface covered with material $i$ to the total planar area.

This generalized Cassie-Wenzel's equation has been presented for macroscopic defects using thermodynamical arguments in Ref. [26]. In Refs. [10, 11], the rigorous proof of this equation has been derived, within a SOS-like model, for microscopic defects covering the surface with a certain periodicity. In the later case the law is satisfied up to a small temperature dependent correction (tending exponentially to zero with the temperature). Namely,

$$
\Delta \tau=r_{1} c_{1}(\Delta \tau)_{1}^{\mathrm{flat}}+r_{2} c_{2}(\Delta \tau)_{2}^{\mathrm{flat}}+O\left(e^{-\beta C}\right)
$$

Let us now consider a surface $z(x, y)$ over a certain area $\mathrm{L} \times \mathrm{L}$ in atomic units. Combining the previous results, we know that we can use the CassieWenzel's equation for defects of order $O(\mathrm{~L})$ or of order $O(1)$. On the other hand, it is also obvious that a real surface can present heterogeneities at all intermediate length-scales $\mathrm{L}^{\varepsilon}$ with $0<\varepsilon<1$. It is thus interesting to extend the proof of the Cassie-Wenzel's relation for such mesoscopic defects $O\left(\mathrm{~L}^{\varepsilon}\right)$, $0<\varepsilon<1$.

This is actually the aim of this paper. We consider an Ising-like lattice gas model with mesoscopic defects. We prove in Theorem 1 below, the validity of the generalized Cassie-Wenzel's equation at low temperatures, within a certain range of the coupling constants. This equation reduces to the Cassie's law when the wall is heterogeneous and flat and to the Wenzel's law when the wall is homogeneous and rough.

Let us stress that contrary to the case of microscopic defects, no corrective term has to be added. 
However, this result is only true when the strength of the interaction between the particles and the wall is small. We give then an important improvement of this law, showing that when this strength is varied, the system exhibits surface phase transitions between two regimes.

Namely, we show in Theorem 2 that, in the homogeneous case, a transition takes place between a Wenzel's and Cassie's behaviours for the drop.

The paper is organized as follows. In Section 2, we introduce the modified semi-infinite Ising model which describes the modeling of the rough and heterogeneous surface, and we give the microscopic definitions of the various surface-tensions. Our results are stated in Section 3.. Finally, Sections 4 and 5 are devoted to proofs.

\section{The model}

To model the influence of roughness and heterogeneities on wetting we use a suitable $3 D$ half-infinite Ising model to describe the drop and its vapor and an SOS surface to represent the boundary of the wall. Namely, we will describe the wall by the boundary $\partial W$ of a half infinite lattice $W \subset \mathbb{Z}^{3}$ which represents the substrate, as shown in Figure 2.

This boundary will be rough (see below for the precise definition of $W$ ) and we shall consider $W$ to be the union of two disjoint subsets $W_{1}$ and $W_{2}$. In this way we get an inhomogeneous wall $\partial W=\partial W_{1} \cup \partial W_{2}$ composed of several pieces of the two different substrates. For the vessel containing the drop and the gas we take the complement $V=\mathbb{Z}^{3} \backslash W$.

To each site $x$ of the vessel $V$, we associate a variable $\sigma_{x}$ which may take two values; +1 associated to a particle at $x$, and -1 associated to an empty site. We assume that the substrate is completely filled, i.e. $\sigma_{x} \equiv+1$ for all $x \in W$.

Inside the vessel, the variables are coupled with a nearest neighbour coupling $J / 2>0$, representing a nearest neighbour attraction of particles while at the boundary between the vessel and the substrate the spins of the vessel are coupled with a nearest neighbour coupling constant, $K_{x} / 2$ with the particles of $W: K_{x}=K_{1}$ or $K_{2}$ according $x \in W_{1}$ or $x \in W_{2}$.

Formally, for any finite set $\Omega \subset V$ these interactions are described by the 
Hamiltonian

$$
\begin{aligned}
H_{\Omega}^{\bar{\sigma}}(\sigma)= & -\frac{J}{2} \sum_{\langle x y\rangle x, y \in \Omega}\left(\sigma_{x} \sigma_{y}-1\right)-\frac{J}{2} \sum_{\langle x y\rangle x \in \Omega, y \in \Omega^{c} \backslash W}\left(\sigma_{x} \bar{\sigma}_{y}-1\right) \\
& -\frac{K_{x}}{2} \sum_{\langle x y\rangle x \in \Omega, y \in W}\left(\sigma_{x}-1\right)
\end{aligned}
$$

Here $\langle x y\rangle$ denotes nearest neighbour pairs, $\Omega^{c}=\mathbb{Z}^{3} \backslash \Omega$ is the complement of $\Omega$, and $\bar{\sigma}$ are the chosen boundary conditions defined as $\bar{\sigma}=+$ or - , i.e. either $\bar{\sigma}_{y}=+1$ for all $y \in \Omega^{c} \backslash W$ or $\bar{\sigma}_{y}=-1$ for all $y \in \Omega^{c} \backslash W$.

Let us now introduce the differential wall tension for the model (2.1). Considering a finite lattice $\Lambda(L)=\left\{\left(x_{1}, x_{2}, x_{3}\right) \in \mathbb{Z}^{3}:\left|x_{i}\right| \leq L, i=1,2,3\right\}$, we let $Z_{W}^{+}(\Omega)$ and $Z_{W}^{-}(\Omega)$ be the partition functions of the model (2.1) at inverse temperature $\beta$, in the volume $\Omega=\Lambda(L) \cap V$, with respectively, + and - boundary conditions on that part of the boundary of $\Lambda(L) \cap V$ which is not part of the wall (on the wall, the boundary conditions are always +1 ). We then define the wall free energy $\tau_{+W}$ (and similarly $\tau_{-W}$ ) in term of $\log Z_{W}^{+}(\Omega)$ by subtracting the bulk term as well as the boundary terms associated with the boundary $\partial \Omega \backslash \partial W$, and taking appropriate limits. The differential wall tension

$$
\Delta \tau=\tau_{+W}-\tau_{-W}
$$

is thus defined as $[16,23,3,2,10]$ :

$$
\beta \Delta \tau=-\lim _{L \rightarrow \infty} \frac{1}{(2 L+1)^{2}} \log \frac{Z_{W}^{-}(\Omega)}{Z_{W}^{+}(\Omega)}
$$

For the usual surface tension $\tau_{+-}$between the + and - phases we use the standard definition [17]. Namely, let $Z^{+}(\Lambda(L))$ be the partition function of the standard Ising model with formal Hamiltonian

$$
-\frac{J}{2} \sum_{\langle x y\rangle}\left(\sigma_{x} \sigma_{y}-1\right)
$$

in the volume $\Lambda(L)$ with + boundary conditions on the boundary of $\Lambda$ and $Z^{+-}(\Lambda(L))$ be the partition function with + boundary conditions below the plane $x_{3}=1 / 2$ and - boundary conditions above this plane. Then, the surface tension $\tau_{+-}$is defined by the limit

$$
\beta \tau_{+-}=-\lim _{L \rightarrow \infty} \frac{1}{(2 L+1)^{2}} \log \frac{Z^{+-}(\Lambda(L))}{Z^{+}(\Lambda(L))}
$$


In the perfectly flat case, the set modeling the substrate will be just the half space $W^{\text {flat }}=\left\{\left(x_{1}, x_{2}, x_{3}\right) \in \mathbb{Z}^{3} \mid x_{3} \leq 0\right\}$ and we let $(\Delta \tau)_{1}^{\text {flat }}$ (resp. $(\Delta \tau)_{2}^{\text {flat }}$ ) correspond to the case of the homogeneous flat wall with $W_{1}=W^{\text {flat }}, W_{2}=\emptyset\left(\right.$ resp. $\left.W_{2}=W^{\text {flat }}, W_{1}=\emptyset\right)$.

More generally, we consider a substrate surface $\partial W$ (defined as the set of unit plaquettes, whose center intersects the bonds $x y, x \in W, y \in \mathbb{Z}^{3} \backslash W$, in their middle point) given by a periodic Solid-On-Solid type interface, i.e. $\partial W$ corresponds to the graph of a periodic function $x_{3}=x_{3}\left(x_{1}, x_{2}\right)$.

For the sake of simplicity, we shall consider a boundary surface $\partial W$ given by the graph of the function $x_{3}\left(x_{1}, x_{2}\right)$ defined on the cylinder $\left\{\frac{1}{2} \leq x_{1} \leq\right.$ $\left.a+\frac{1}{2}, \frac{1}{2} \leq x_{2} \leq a+\frac{1}{2}\right\}$ by

$$
x_{3}\left(x_{1}, x_{2}\right)=\left\{\begin{array}{cl}
-b+\frac{1}{2} & \text { for } \frac{1}{2} \leq x_{1} \leq c+\frac{1}{2}, \frac{1}{2} \leq x_{2} \leq c+\frac{1}{2} \\
\frac{1}{2} \text { otherwise } & \text { otherise }
\end{array}\right.
$$

and determined on the complement of this cylinder by the periodicity (see Figure 2).

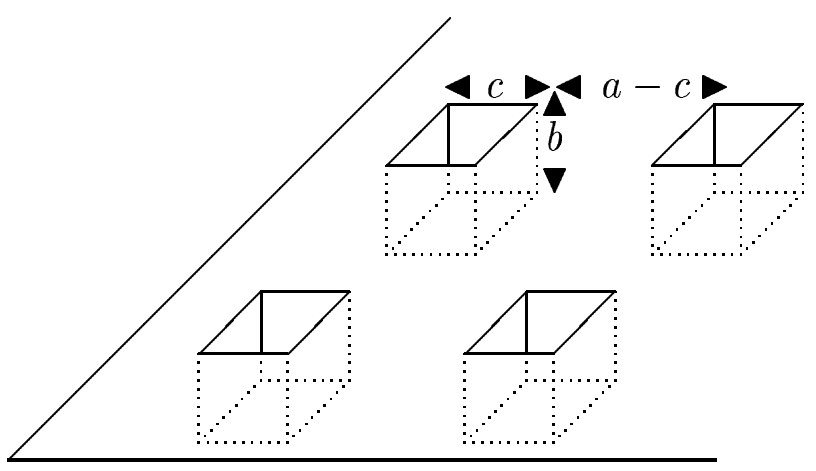

Figure 2: The substrate surface $\partial W$.

We take a mesoscopic length-scale for the size of the pores. Namely, we choose $a=a_{0} f(L), b=b_{0} f(L), c=c_{0} f(L), d=d_{0} f(L)$, where $\lim _{L \rightarrow \infty} f(L)$ $=\infty$ and $\lim _{L \rightarrow \infty} f(L) / L=0$. The roughness of the wall is $r=\lim _{L \rightarrow+\infty}(1+$ $\left.4 b c / a^{2}\right)=1+4 b_{0} c_{0} / a_{0}^{2}$.

Finally to describe heterogeneities, we take $W_{1}$ as the part of the wall $W$ below the plane $x_{3}=-d+1 / 2$ and $W_{2}$ as the part of $W$ above this plane $(0 \leq d \leq b)$. 
We use $A_{1}$ and $A_{2}$ to denote the area of the substrate surfaces $\partial W_{1}$ and $\partial W_{2}$ and $\bar{A}_{1}$ and $\bar{A}_{2}$ their projection onto the horizontal plane. The respective roughness $r_{1}, r_{2}$ and concentrations $c_{1}, c_{2}$, can then be defined by

$$
r_{k}=\frac{A_{k}}{\bar{A}_{k}}, \quad c_{k}=\frac{\bar{A}_{k}}{\bar{A}_{1}+\bar{A}_{2}}, \quad k=1,2
$$

in terms of which the roughness reads $r=r_{1} c_{1}+r_{2} c_{2}$.

\section{Results}

Our first result establishes the validity of the generalized Cassie-Wenzel's equation for the model defined in the previous section.

Theorem 1 Assume that the parameters introduced above satisfy the conditions

$$
\begin{aligned}
C \equiv & J\left[1-\max \left(\frac{1}{2}, \frac{r+c_{1}-1}{r+2 c_{1}-1}\right)\right] \\
& -\left|K_{1}\right| \frac{r_{1}}{r_{1}+1}-\left|K_{2}\right| \max \left(\frac{1}{2}, \frac{r_{2} c_{2}-c_{2}}{r_{2} c_{2}-c_{2}+c_{2}-1}\right)>0
\end{aligned}
$$

and that the temperature is sufficiently low, namely $\beta C>5.71$, then

$$
\Delta \tau=r_{1} c_{1}(\Delta \tau)_{1}^{\text {flat }}+r_{2} c_{2}(\Delta \tau)_{2}^{\text {flat }}
$$

The condition (3.1) (which can be viewed as a condition of smallness of $\left|K_{1}\right| / J$ and $\left.\left|K_{2}\right| / J\right)$ ensures that the configurations + and - are the respective ground states of $H^{+}$and $H^{-}: \min _{\sigma} H_{\Omega}^{+}(\sigma) \geq H_{\Omega}^{+}(-)$and $\min _{\sigma} H_{\Omega}^{-}(\sigma) \geq$ $H_{\Omega}^{-}(-)$. Let $h^{ \pm}(\sigma)=\lim _{L \rightarrow \infty} \frac{H_{\Omega}^{ \pm}(\sigma)}{(2 L+1)^{2}}$ be the specific energies per unit surface. One has $h^{+}(+)=0$ and $h^{-}(-)=r_{1} c_{1} K_{1}+r_{2} c_{2} K_{2}$. This implies that the law (3.2) holds true at the level of ground states. has

Indeed, letting $\Delta e=\lim _{L \rightarrow \infty} \frac{1}{(2 L+1)^{2}}\left[\min _{\sigma} H_{\Omega}^{-}(\sigma)-\min _{\sigma} H_{\Omega}^{+}(\sigma)\right]$, one

$$
\Delta e=r_{1} c_{1} K_{1}+r_{2} c_{2} K_{2}
$$

The proof of this result at the level of free energies is given in Section 4. Let us mention the study on Cassie's law proposed in [15] whose results do not rely on the knowledge of ground states.

Our second result concerns the homogeneous case. We will assume that $K_{1}=K_{2}$. We let $\rho=1+4 b_{0} / c_{0}$ be the relative roughness of the pores and let $c^{\prime}=\left(c_{0} / a_{0}\right)^{2}$ be the density of the pores. 
Theorem 2 i) If $-J / \rho<K<J / \rho$, then

$$
\Delta \tau=r(\Delta \tau)^{f l a t}
$$

ii) If $J / \rho<K<J$, then

$$
\Delta \tau=c^{\prime} \tau_{+-}+\left(1-c^{\prime}\right)(\Delta \tau)^{f a t}
$$

iii) If $-J<K<-J / \rho$, then

$$
\Delta \tau=-c^{\prime} \tau_{+-}+\left(1-c^{\prime}\right)(\Delta \tau)^{f a t}
$$

As before, it is assumed that the temperature is sufficiently low, see (5.2) and (5.52).

Let us here stress the physical meaning of these results.

According to the relative strength of the solid/liquid $(K)$ and the liquid/gaz $(J)$ interactions, the system will mimic one of the ground states corresponding to situations where either the liquid fills the pores of the substrate, or leaves these pores empty (see below). In the first case we recover the Wenzel's law (3.5) that, according to macroscopic considerations, governs the behaviour of a sessile drop of liquid sitting on top of a rough and homogeneous wall. However, from these microscopic considerations, we get that a sufficient enhancing of the affinity between the liquid and the gas phase gives rise to a Cassie-type behaviour due to the additional liquid/gaz interfaces created by the absence of liquid within the pores. To see that this difference is a quantitative one, let's consider for the sake of definiteness a drop of water on top of a polyethylene therephtalate (PET) surface. The wall energy $(\Delta \tau)^{\text {flat }}$ of PET is $40 \mathrm{mN} / \mathrm{m}$, and the superficial tension $\tau$ of water is $72.4 \mathrm{mN} / \mathrm{m}$. We thus have :

$$
\left.\cos \theta\right|_{\text {Wenzel }}=\frac{40}{72.4} \frac{1}{r}
$$

to be compared to :

$$
\left.\cos \theta\right|_{\text {Cassie }}=\frac{40}{72.4}-c^{\prime}\left(1+\frac{40}{72.4}\right)
$$

A typical roughness for such a surface is 1.5 . We thus get that versus $c^{\prime}$, the cosine of the equilibrium contact angle $\theta$ behaves as depicted in Figure 3: 


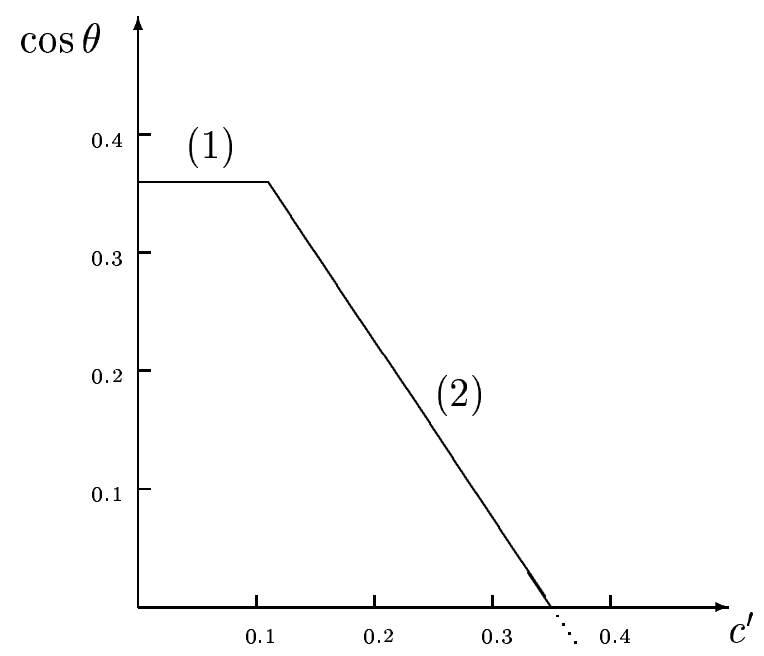

Figure 3: Dependence of the equilibrium contact angle $\theta$ on the "density of pores" $c^{\prime}$ exhibiting a transition between a Wenzel's regime (1) and a Cassie's regime (2).

Let us emphasize that this result, if it can be carried over to real surfaces, suggests that the wetting properties of a rough wall are not only driven by the roughness $r$ of the wall, but do also depend on the particular geometry that gives rise to $r$.

We end this section with the ground states description in the homogeneous case. Let $\sigma_{k}^{+}$be the configuration with all + above the plane $x_{3}=k+1 / 2 \quad(k \geq 0)$ and all - below this plane. It is easy to check that these configurations together with the configuration with all + minimize the Hamiltonian with + boundary conditions. One has $\min _{\sigma} H_{\Omega}^{+}(\sigma) \geq$ $\min _{\sigma \in\left\{+, \sigma_{k}^{+}\right\}} H_{\Omega}^{+}(\sigma)$ and the specific energy $h^{+}(\sigma)$ takes the following values:

$$
\begin{aligned}
h^{+}(+) & =0 \\
h^{+}\left(\sigma_{0}^{+}\right) & =\frac{c^{2}}{a^{2}} J+\frac{c^{2}+4 b c}{a^{2}} K \\
h^{+}\left(\sigma_{k}^{+}\right) & =J+r K \quad \text { for all finite } \quad k \geq 1
\end{aligned}
$$

Notice that $h^{+}(+)=h^{+}\left(\sigma_{0}^{+}\right)$on the line $K=-J / \rho$ and $h^{+}\left(\sigma_{0}^{+}\right)=h\left(\sigma_{k}^{+}\right)$on the line $K=-J$. Analogously, let $\sigma_{k}^{-}$be the configuration with all - above the plane $x_{3}=k+1 / 2$ and all + below this plane. 
One has $\min _{\sigma} H_{\Omega}^{-}(\sigma) \geq \min _{\sigma \in\left\{-, \sigma_{k}^{-}\right\}} H_{\Omega}^{-}(\sigma)$ and:

$$
\begin{aligned}
h^{-}(-) & =r K \\
h^{-}\left(\sigma_{0}^{-}\right) & =\frac{c^{2}}{a^{2}} J+\frac{a^{2}-c^{2}}{a^{2}} K \\
h^{-}\left(\sigma_{k}^{-}\right) & =J \quad \text { for all finite } \quad k \geq 1
\end{aligned}
$$

Notice that $h^{-}(-)=h^{-}\left(\sigma_{0}^{-}\right)$on the line $K=J / \rho$ and $h^{-}\left(\sigma_{0}^{-}\right)=h^{-}\left(\sigma_{k}^{-}\right)$on the line $K=J$.

The formulae (3.7) and (3.8) lead to the phase diagram shown in Figure 4.

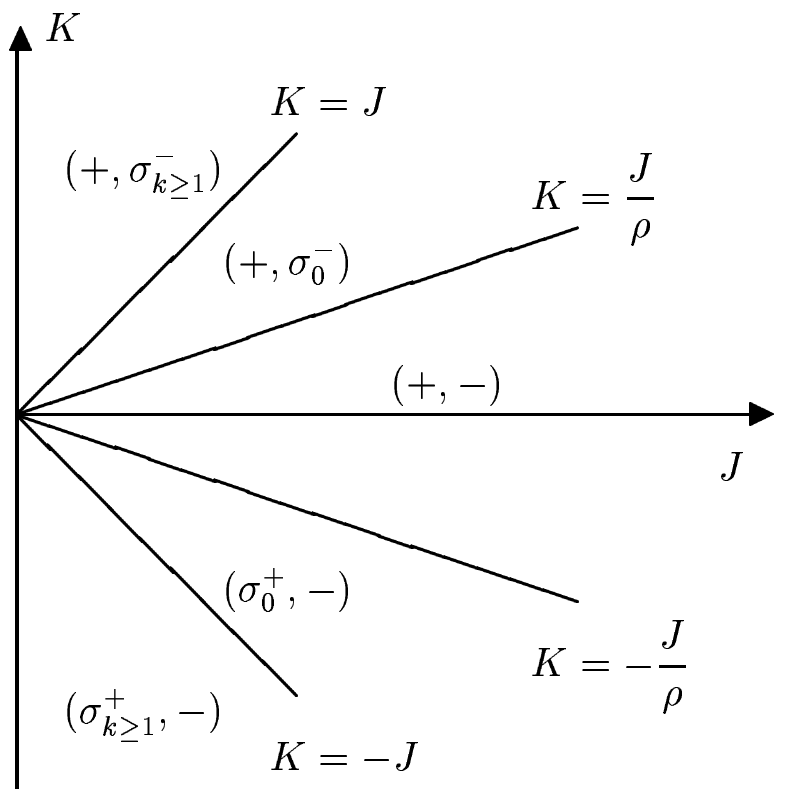

Figure 4: The diagram of ground states.

They show that the results (3.4-3.6) hold true at the level of ground state. Indeed,

$$
\Delta e=r K
$$

when $-J / \rho<K<J / \rho$,

$$
\Delta e=c^{\prime} J+\left(1-c^{\prime}\right) K
$$


when $J / \rho<K<J$, and finally

$$
\Delta e=-c^{\prime} J+\left(1-c^{\prime}\right) K
$$

when $-J<K<-J / \rho$.

The proof of these results at the level of free energies is given in Section 5 .

\section{Proof of Theorem 1}

To prove the result at the level of free energies, we have to take into account the excitations of ground states. To this end we begin with a contour representation of partition functions $Z_{W}^{+}(\Omega)$ and $Z_{W}^{-}(\Omega)$. A natural definition is to consider the contours as boundaries of regions where the considered configuration differs from the corresponding ground state configuration.

For $Z_{W}^{+}(\Omega)$ we have a standard representation introducing for any configuration $\sigma$ (such that $\sigma_{x}=+1$ for all $x \in \Omega^{c}$ ) the contours as connected components of the set $B^{+}(\sigma)$ of all plaquettes of the dual lattice that separate two neighbouring sites $x, y \in V$ with $\sigma_{x} \neq \sigma_{y}$.

For any contour $\gamma$ we introduce the weight factor

$$
z^{+}(\gamma)=e^{-\beta\left(J\left|\gamma_{\mathbf{b k}}\right|+K_{1}\left|\gamma_{W_{1}}\right|+K_{2}\left|\gamma_{W_{2}}\right|\right)}
$$

Here we define $\gamma_{W_{i}}=\gamma \cap \partial W_{i}, i=1,2$, and $\gamma_{\mathrm{bk}}=\gamma \backslash\left(\gamma_{W_{1}} \cup \gamma_{W_{2}}\right) ;\left|\gamma_{\mathrm{bk}}\right|$, $\left|\gamma_{W_{i}}\right|$, is the number of plaquettes of $\gamma_{\mathrm{bk}}, \gamma_{W_{i}}$, respectively. In terms of the weight factors $z^{+}(\gamma)$ one clearly has

$$
Z_{W}^{+}(\Omega)=\sum_{\left\{\gamma_{1}, \ldots, \gamma_{n}\right\}_{\mathrm{comp}}} \prod_{i=1}^{n} z^{+}\left(\gamma_{i}\right)
$$

where $\left\{\gamma_{1}, \ldots, \gamma_{n}\right\}_{\text {comp }}$ is a collection of compatible (mutually disjoint) contours in $\Omega$.

To get a similar expression for $Z_{W}^{-}(\Omega)$, we only have to be careful with the definition of contours touching the wall. Namely, for configurations $\sigma$ such that $\sigma_{x}=+1$ for $x \in W$ and $\sigma_{x}=-1$ for $x \in \Omega^{c} \backslash W$, we introduce contours as connected component of the set $B^{-}(\sigma)$ of all plaquettes separating nearest neighbour sites $x, y \in V$ for which $\sigma_{x} \neq \sigma_{y}$ or nearest neighbour sites $x \in$ $V, y \in W$ for which $\sigma_{x}=\sigma_{y}(=+1)$. Introducing now the weight $z^{-}(\gamma)$ as

$$
z^{-}(\gamma)=e^{-\beta\left(J\left|\gamma_{\mathbf{b k}}\right|-K_{1}\left|\gamma_{W_{1}}\right|-K_{2}\left|\gamma_{W_{2}}\right|\right)}
$$


we get

$$
Z_{W}^{-}(\Omega)=e^{-\beta K_{1} A_{1}(\Omega)-\beta K_{2} A_{2}(\Omega)} \sum_{\left\{\gamma_{1}, \ldots, \gamma_{n}\right\}_{\text {comp }}} \prod_{i=1}^{n} z^{-}\left(\gamma_{i}\right)
$$

where $A_{i}(\Omega)$ is the number of bonds $x y, x \in \Omega, y \in W_{i}$. Notice that the set of contours in both situations exactly coincide (even though the weights do not) and the sums in (4.2) and (4.4) are over exactly the same collections of contours. Notice also that the weights (4.1) (4.3) differs only if $\gamma$ touches the wall i.e., if $\gamma \cap \partial W \neq \emptyset$.

To be able to control, in terms of convergent cluster expansions, $\ln Z_{W}^{+}(\Omega)$ and $\ln Z_{W}^{-}(\Omega)$, the weights $z^{+}(\gamma)$ and $z^{-}(\gamma)$ must satisfy the dumping condition $\left|z^{ \pm}(\gamma)\right| \leq e^{-\lambda|\gamma|}$, where $\lambda$ is a fixed sufficiently large constant and $|\gamma|=\left|\gamma_{\mathrm{bk}}\right|+\left|\gamma_{W_{1}}\right|+\left|\gamma_{W_{2}}\right|$. To find upper bounds for $\left|z^{-}(\gamma)\right|$ and $\left|z^{+}(\gamma)\right|$ we notice that

$$
\begin{aligned}
J\left|\gamma_{\mathrm{bk}}\right| & -\left|K_{1}\right|\left|\gamma_{W_{1}}\right|-\left|K_{2}\right|\left|\gamma_{W_{2}}\right| \\
& =\left[J-J \frac{\left|\gamma_{W_{1}}\right|+\left|\gamma_{W_{2}}\right|}{|\gamma|}-\left|K_{1}\right| \frac{\left|\gamma_{W_{1}}\right|}{|\gamma|}-\left|K_{2}\right| \frac{\left|\gamma_{W_{2}}\right|}{|\gamma|}\right]|\gamma|
\end{aligned}
$$

Realizing by easy geometrical observations that the term inside brackets is greater than $C$, one gets by the definitions (4.1) (4.3):

$$
\left|z^{ \pm}(\gamma)\right| \leq e^{-\beta C|\gamma|}
$$

We now introduce multi-indexes in order to write the logarithm of the partition functions $Z_{W}^{+}(\Omega)$ and $Z_{W}^{-}(\Omega)$ as a sum over these multi-indexes (see [20]). A multi-index $X$ is a function from the set of contours into the set of non negative integers, and we let $\operatorname{supp} X=\{\gamma: X(\gamma) \geq 1\}$. We define the truncated functionals

$$
\Phi^{ \pm}(X)=\frac{a(X)}{\prod_{\gamma} X(\gamma) !} \prod_{\gamma} z^{ \pm}(\gamma)^{X(\gamma)}
$$

where the factor $a(X)$ is a combinatoric factor defined in terms of the connectivity properties of the graph $G(X)$ with vertices corresponding to $\gamma \in$ supp $X$ (there are $X(\gamma)$ vertices for each $\gamma \in \operatorname{supp} X$ ) that are connected by an edge whenever the corresponding contours are incompatible). Namely, $a(X)=0$ and hence $\Phi^{ \pm}(X)=0$ unless $G(X)$ is a connected graph and

$$
a(X)=\sum_{G \subset G(X)}(-1)^{|e(G)|}
$$


Here the sum goes over connected subgraphs $G$ whose vertices coincide with the vertices of $G(X)$ and $|e(G)|$ is the number of edges of the graph $G$. If the cluster $C$ contains only one contour, then $a(\gamma)=1$.

The standard cluster expansion [17] [13] [20], then yields

$$
\ln \sum_{\left\{\gamma_{1}, \ldots, \gamma_{n}\right\}_{\text {comp }}} \prod_{i=1}^{n} z^{ \pm}(\gamma)=\sum_{X \in \chi(\Omega)} \Phi^{ \pm}(X)
$$

Here $\chi(\Omega)$ is the set of all multi-indexes $X$ having all contours in $\Omega$.

The convergence of the cluster expansion holds, c.f. [13] [20], as soon as one can find a positive real-valued function $\mu(\gamma)$ such that

$$
z(\gamma) \exp \left\{-\sum_{\gamma^{\prime} \nsim \gamma} \mu\left(\gamma^{\prime}\right)\right\} \leq \mu(\gamma)
$$

Here the sum runs over contours $\gamma^{\prime}$ incompatible with $\gamma$ : this relation is denoted by $\gamma^{\prime} \nsim \gamma$ and means that $\gamma^{\prime}$ intersects $\gamma$. Taking into account that the number contours $\gamma$ of size $\ell$ passing to a given point is less then $12^{2 \ell}$, the area of contours is even, with minimal value $\left|\gamma^{\mathrm{min}}\right|=6$, that $\sum_{\gamma^{\prime} \nsim \gamma} \mu\left(\gamma^{\prime}\right) \leq|\gamma| \sum_{\gamma^{\prime} \ni p} \mu\left(\gamma^{\prime}\right)$, and choosing $\mu(\gamma)=\left(12^{2} e^{t}\right)^{-|\gamma|}$, the above convergence condition will be satisfied here whenever

$$
\beta C>\ln 12^{2}+t+\frac{e^{-6 t}}{1-e^{-2 t}} \geq 5.71
$$

It implies

$$
\sum_{X: X(\gamma) \geq 1}\left|\Phi^{ \pm}(X)\right| \leq \mu(\gamma)
$$

As a result of (4.8) we can write

$$
\ln Z_{W}^{+}(\Omega)-\ln Z_{W}^{-}(\Omega)-\beta K_{1} A_{1}-\beta K_{2} A_{2}=\sum_{X \in \chi(\Omega)}\left[\Phi^{+}(X)-\Phi^{-}(X)\right]
$$

By definitions (4.1) and (4.3) the contributions of the contours in the bulk are exactly the same for the + or - b.c. Thus all terms with $X$ supported by contours not touching the wall are canceled in the above difference of the logarithms and only the sum over $X$ containing contours touching the wall remains. We use $\chi_{W}(\Omega)$ to denote the set of all such multi-indexes $X$. Then,

$$
\ln Z_{W}^{+}(\Omega)-\ln Z_{W}^{-}(\Omega)-\beta K_{1} A_{1}-\beta K_{2} A_{2}=\sum_{X \in \chi_{W}(\Omega)}\left[\Phi^{+}(X)-\Phi^{-}(X)\right]
$$


Using the fact that $z^{ \pm}(\gamma)$ are invariant under horizontal translation by multiples of the periodicity constant $a$ and satisfy the bound (4.5), one get,

$$
\begin{aligned}
\Delta \tau-r_{1} c_{1} K_{1}-r_{2} c_{2} K_{2} & =\lim _{L \rightarrow \infty} \frac{1}{\beta(2 L+1)^{2}} \sum_{X \in \chi_{W}(\Omega)}\left[\Phi^{+}(X)-\Phi^{-}(X)\right] \\
& =\lim _{a \rightarrow \infty} \frac{1}{\beta a^{2}} \sum_{X \in \chi_{W}\left(\Omega_{a}\right)}\left[\Phi^{+}(X)-\Phi^{-}(X)\right]
\end{aligned}
$$

where $\Omega_{a}=V \cap \Lambda_{a}$, with

$$
\Lambda_{a}=\left\{x \in \mathbb{Z}^{3}: 0 \leq x_{1} \leq a, 0 \leq x_{2} \leq a,\left|x_{3}\right| \leq a\right\}
$$

Let us now turn to the flat walls. Let $\Omega^{\prime}$ be a box in the semi-infinite lattice

$$
\mathbb{L}=\left\{\left(x_{1}, x_{2}, x_{3}\right) \in \mathbb{Z}^{3}: x_{3}>0\right\}
$$

and let $\Pi=\partial W^{\text {flat }}$ be the plane $x_{3}=1 / 2$. We let $Z_{W_{1}^{\text {flat }}}^{ \pm}\left(\Omega^{\prime}\right)$ and $Z_{W_{2}^{\text {flat }}}^{ \pm}\left(\Omega^{\prime}\right)$ be the partition functions corresponding to the case of the flat walls. We define the contours as before and introduce the weights

$$
z_{j}^{ \pm}(\gamma)=e^{-\beta J\left(\left|\gamma_{\mathrm{bk}}\right| \pm K_{j}\left|\gamma_{\mathrm{f}}\right|\right)}
$$

Here $\gamma_{\mathrm{f}}=\gamma \cap \Pi$ and $\gamma_{\mathrm{bk}}=\gamma \backslash \gamma_{\mathrm{f}}$; we say that $\gamma$ touches $\Pi$ if it contains plaquettes of this plane. Then,

$$
\begin{aligned}
Z_{W_{i}^{\text {flat }}}^{+}\left(\Omega^{\prime}\right) & =\sum_{\left\{\gamma_{1}, \ldots, \gamma_{n}\right\}_{\mathrm{comp}}} \prod_{i=1}^{n} z_{j}^{+}\left(\gamma_{i}\right) \\
Z_{W_{i}^{\text {flat }}}^{-}\left(\Omega^{\prime}\right) & =e^{-\beta K_{i} A\left(\Omega^{\prime}\right)} \sum_{\left\{\gamma_{1}, \ldots, \gamma_{n}\right\}_{\mathrm{comp}}} \prod_{i=1}^{n} z_{j}^{-}\left(\gamma_{i}\right)
\end{aligned}
$$

Here $\left\{\gamma_{1}, \ldots, \gamma_{n}\right\}_{\text {comp }}$ are collections of compatible contours in $\Omega^{\prime}$ and $A\left(\Omega^{\prime}\right)$ is the number of bonds $x y, x \in \Omega^{\prime}, y \in \mathbb{Z}^{3} \backslash \Omega^{\prime}$, that cross the plane $\Pi$. We let $\Phi_{i}^{ \pm}$be the truncated functional associated to the weights (4.14). Then

$$
\ln Z_{W_{i}^{\text {flat }}}^{+}\left(\Omega^{\prime}\right)-\ln Z_{W_{i}^{\text {flat }}}^{-}\left(\Omega^{\prime}\right)-\beta K_{i} A\left(\Omega^{\prime}\right)=\sum_{X \in \chi_{\Pi}\left(\Omega^{\prime}\right)}\left[\Phi_{i}^{+}(X)-\Phi_{i}^{-}(X)\right]
$$


where $\chi_{\Pi}\left(\Omega^{\prime}\right)$ is the set of multi-indexes of $\chi\left(\Omega^{\prime}\right)$ whose support intersect the plane $\Pi$. Using that the weights are now completely invariant with respect to horizontal translations, we have

$$
\begin{aligned}
(\Delta \tau)_{1}^{\mathrm{flat}}-K_{1} & =\lim _{L \rightarrow \infty} \frac{1}{\beta a^{2}} \sum_{X \in \chi_{\Pi}\left(\Omega_{a}^{\prime}\right)}\left[\Phi_{1}^{+}(X)-\Phi_{1}^{-}(X)\right] \\
& =\sum_{X \in \chi_{\Pi}(\mathbb{L}) p \in X} \frac{\left[\Phi_{1}^{+}(X)-\Phi_{1}^{-}(X)\right]}{|X \cap \Pi|} \equiv \mathcal{F}_{1}
\end{aligned}
$$

and

$$
\begin{aligned}
(\Delta \tau)_{2}^{\mathrm{flat}}-K_{2} & =\lim _{L \rightarrow \infty} \frac{1}{\beta a^{2}} \sum_{X \in \chi_{\Pi}\left(\Omega_{a}^{\prime}\right)}\left[\Phi_{2}^{+}(X)-\Phi_{2}^{-}(X)\right] \\
& =\sum_{X \in \chi_{\Pi}(\mathbb{L}) p \in X} \frac{\left[\Phi_{2}^{+}(X)-\Phi_{2}^{-}(X)\right]}{|X \cap \Pi|} \equiv \mathcal{F}_{2}
\end{aligned}
$$

Here $\Omega_{a}^{\prime}=\Lambda_{a} \cap \mathbb{L}$ and the two last sums in (4.18) (4.19) are over multi-indexes whose support contains a given plaquette of the plane $\Pi$.

Our last step is to compare the R.H.S. of (4.13) with (4.18) and (4.19). To this end, we split the sum over multi-indexes $X \in \chi_{\Pi_{W}}\left(\Omega_{a}\right)$ in three terms $S_{1}(a), S_{2}(a)$, and $R(a)$. The first term $S_{1}(a)$ is the sum over $X$ that intersect only one face of the part $\left(\partial W_{1}\right)_{a}$ of the boundary of the wall that separates $W_{1}$ from $\Omega_{a}$. Notice that for the multi-indexes $X$ involved in this sum, one has $\Phi^{+}(X)=\Phi_{1}^{+}(X)$. Furthermore, since $\left(\partial W_{1}\right)_{a}$ has five faces, $S_{1}(a)$ is the sum of five terms and each of them divided by the area of corresponding face will actually equal $\mathcal{F}_{1}$ in the limit $a \rightarrow \infty$. Thus

$$
\lim _{a \rightarrow \infty} S_{1}(a) / \beta a^{2}=r_{1} c_{1} \mathcal{F}_{1}
$$

The second term $S_{2}(a)$ is the sum over multi-indexes that intersect only one face of the part $\left(\partial W_{2}\right)_{a}$ of the boundary of the wall that separates $W_{2}$ from $\Omega_{a}$. In that case $\Phi^{ \pm}(X)=\Phi_{2}^{ \pm}(X)$ and we get analogously to the previous situation

$$
\lim _{a \rightarrow \infty} S_{2}(a) / \beta a^{2}=r_{2} c_{2} \mathcal{F}_{2}
$$

Finally, the reminder $R(a)$ contains the terms where the supports of multiindexes intersect at least two faces of $(\partial W)_{a}=\left(\partial W_{1}\right)_{a} \cup\left(\partial W_{2}\right)_{a}$. It thus 
can be bounded by a constant times the length of the boundary of faces (for the adjacent ones) plus a term proportional to the area of the vertical faces times a negative exponential small correction with a power proportional to the length between the opposed faces. Thus the ratio $R(a) / \beta a^{2}$ goes to 0 as $a$ goes to infinity and we get

$$
\Delta \tau-r_{1} c_{1} K_{1}-r_{2} c_{2} K_{2}=r_{1} c_{1} \mathcal{F}_{1}+r_{2} c_{2} \mathcal{F}_{2}
$$

giving the desired result.

\section{Proof of Theorem 2}

We first consider the proof of Wenzel's regime stated in (3.4) when $|K|<J / \rho$. In this situation, the condition on the parameters $K$ and $J$ ensures that the configurations + and - are the respective ground states of $H^{+}$and $H^{-}$. Here, one has simply to notice that for the homogeneous wall the weights of contours satisfy

$$
\left|z^{ \pm}(\gamma)\right| \leq e^{-\beta\left(\frac{J-\rho|K|}{\rho+1}\right)|\gamma|}
$$

The situation then turns out to be a particular case of the problem already analyzed in Section 4. The needed condition on the temperature is

$$
\beta \frac{J-\rho|K|}{\rho+1}>5.71
$$

We now turn to the proof of Cassie's regime stated in (3.5) assuming that $J / \rho<K<J$

\subsection{The flat differential tension $(\Delta \tau)^{\text {flat }}$}

Let us first consider the partitions functions $Z_{W^{\text {flat }}}^{ \pm}\left(\Omega^{\prime}\right)$ corresponding to a flat wall in a box $\Omega^{\prime} \subset \mathbb{L}$. For the partition function $Z_{W^{\text {flat }}}^{-}\left(\Omega^{\prime}\right)$ we define the contours (as in Section 4) as connected component of the set $B^{-}(\sigma)$ of all plaquettes separating nearest neighbour sites $x, y$ for which $\sigma_{x} \neq \sigma_{y}$ if the bond $x y$ does not cross the plane $\Pi$ or nearest neighbour sites $x, y$ for which $\sigma_{x}=\sigma_{y}$ if the bond $x y$ crosses the plane $\Pi$. We introduce the weights

$$
z_{\mathrm{f}}^{-}(\gamma)=e^{-\beta\left(J\left|\gamma_{\mathbf{b k}}\right|-K\left|\gamma_{\mathbf{f}}\right|\right)}
$$


where $\gamma_{\mathrm{f}}=\gamma \cap \Pi$ and $\gamma_{\mathrm{bk}}=\gamma \backslash \gamma_{\mathrm{f}}$. In term of these weights, one has

$$
Z_{W^{\text {flat }}}^{-}\left(\Omega^{\prime}\right)=e^{-\beta K A\left(\Omega^{\prime}\right)} \sum_{\left\{\gamma_{1}, \ldots, \gamma_{n}\right\}_{\mathrm{comp}}} \prod_{i=1}^{n} z_{\mathrm{f}}^{-}\left(\gamma_{i}\right)
$$

where $\left\{\gamma_{1}, \ldots, \gamma_{n}\right\}_{\text {comp }}$ are families of compatible contours in $\Omega^{\prime}$ and $A\left(\Omega^{\prime}\right)$ is the number of bonds $x y, x \in \Omega^{\prime}, y \in \mathbb{Z}^{3} \backslash \Omega^{\prime}$, that crosses the plane $\Pi$. For the partition function $Z_{W}^{+}$flat $\left(\Omega^{\prime}\right)$ we use the standard definition of contours and introduce the weights

$$
z_{\mathrm{f}}^{+}(\gamma)=e^{-\beta\left(J\left|\gamma_{\mathrm{bk}}\right|+K\left|\gamma_{\mathrm{f}}\right|\right)}
$$

to get

$$
Z_{W^{\text {flat }}}^{+}\left(\Omega^{\prime}\right)=\sum_{\left\{\gamma_{1}, \ldots, \gamma_{n}\right\}_{\mathrm{comp}}} \prod_{i=1}^{n} z_{\mathrm{f}}^{+}\left(\gamma_{i}\right)=\exp \left(\sum_{X \in \chi\left(\Omega^{\prime}\right)} \widetilde{\Phi}_{\mathrm{f}}^{+}(X)\right)
$$

where $\widetilde{\Phi}_{\mathrm{f}}^{+}$is the truncated functional corresponding to $z_{\mathrm{f}}^{+}$. It will be convenient to sum over the multi-indexes with same support. We thus introduce the functional

$$
\Phi_{\mathrm{f}}^{+}(S)=\sum_{X: \operatorname{supp} X=S} \widetilde{\Phi}_{\mathrm{f}}^{+}(X)
$$

to get

$$
Z_{W^{\text {flat }}}^{+}\left(\Omega^{\prime}\right)=\sum_{\left\{\gamma_{1}, \ldots, \gamma_{n}\right\}_{\text {comp }}} \prod_{i=1}^{n} z_{\mathrm{f}}^{+}\left(\gamma_{i}\right)=\exp \left(\sum_{S \in \chi\left(\Omega^{\prime}\right)} \Phi_{\mathrm{f}}^{+}(S)\right)
$$

where (with an abuse of notation) $\chi\left(\Omega^{\prime}\right)$ denote the set of the supports of multi-indexes in $\Omega$. The supports of multi-indexes will be called clusters.

Since by definitions the weights of contours are the same for contours not touching the plane $\Pi$, we have

$$
Z_{W^{\text {flat }}}^{-}\left(\Omega^{\prime}\right)=e^{-\beta K A\left(\Omega^{\prime}\right)} \sum_{\substack{\left\{\gamma_{1}, \ldots, \gamma_{n}\right\}_{\text {comp }} \\ \gamma_{i} \cap \Pi \neq \emptyset}} \prod_{i=1}^{n} z_{\mathrm{f}}^{-}\left(\gamma_{i}\right) \sum_{\substack{\left\{\gamma_{1}^{\prime}, \ldots, \gamma_{m}^{\prime}\right\}_{\text {comp }} \\ \gamma_{j}^{\prime} \cap \Pi=\emptyset, \gamma_{j}^{\prime} \sim \gamma_{i}}} \prod_{i=1}^{m} z_{\mathrm{f}}^{+}\left(\gamma_{j}^{\prime}\right)
$$

Here the first sum is over (compatible) families of contours touching the plane $\Pi$ and the second ones is over (compatible) families of contours not touching 
the plane $\Pi$ and compatible with the first family. From relations (5.8-5.9) one has

$$
\begin{aligned}
\frac{Z_{W_{\text {flat }}}^{-}\left(\Omega^{\prime}\right)}{Z_{W^{\text {flat }}}^{+}\left(\Omega^{\prime}\right)} & =e^{-\beta K A\left(\Omega^{\prime}\right)} \sum_{\substack{\left\{\gamma_{1}, \ldots, \gamma_{n}\right\} \text { comp } \\
\gamma_{i} \cap \Pi \neq \emptyset}} \prod_{i=1}^{n} z_{\mathrm{f}}^{-}\left(\gamma_{i}\right) \exp \left(-\sum_{\substack{S: S \in \chi_{\Pi}\left(\Omega^{\prime}\right) \\
\text { orS } S \gamma_{i}}} \Phi_{\mathrm{f}}^{+}(S)\right) \\
& =e^{-\beta K A\left(\Omega^{\prime}\right)} \sum_{\substack{\left\{\gamma_{1}, \ldots, \gamma_{n}\right\} \operatorname{comp} \\
\gamma_{i} \cap \Pi \neq \emptyset}} \prod_{i=1}^{n} z_{\mathrm{f}}^{-}\left(\gamma_{i}\right) \prod_{\substack{S: S \in \chi_{\Pi}\left(\Omega^{\prime}\right) \\
\text { or } S \nsim \gamma_{i}}} e^{-\Phi_{\mathrm{f}}^{+}(S)}
\end{aligned}
$$

where the sum in the exponential and in the last product are over clusters $S$ touching the plane $\Pi$ or incompatible with some contour $\gamma_{i}$ of the family $\left\{\gamma_{1}, \ldots, \gamma_{n}\right\}$ (the relation denoted $S \nsim \gamma_{i}$ means that $S$ intersects $\gamma_{i}$ ), or both. To expand this product, we define the aggregates $A$ as connected families of clusters. Introducing the weights

$$
\widetilde{\rho}_{\mathrm{f}}(A)=\prod_{S \in A} e^{-\Phi_{\mathrm{f}}^{+}(S)}-1
$$

we get

$$
\frac{Z_{W_{\text {flat }}^{\text {fat }}}^{-}\left(\Omega^{\prime}\right)}{Z_{W^{\text {flat }}}^{+}\left(\Omega^{\prime}\right)}=e^{-\beta K A\left(\Omega^{\prime}\right)} \sum_{\substack{\left\{\gamma_{1}, \ldots, \gamma_{n}\right\}_{\text {comp }} \\ \gamma_{i} \cap \Pi \neq \emptyset}} \prod_{i=1}^{n} z_{\mathrm{f}}^{-}\left(\gamma_{i}\right) \sum_{\substack{\left\{A_{1}, \ldots, A_{m}\right\}_{\text {comp }} \\ A_{j} \cap \Pi \neq \emptyset \text { or } \cap A_{j} \nsim \gamma_{i}}} \prod_{j=1}^{m} \widetilde{\rho}_{\mathrm{f}}\left(A_{j}\right)
$$

where the second sum is over families of aggregates touching the wall or incompatible with a contour of the family $\left\{\gamma_{1}, \ldots, \gamma_{n}\right\}$.

As it was done for multi-indexes, it is convenient to sum over all aggregates with the same support. We define the weight

$$
\rho_{\mathrm{f}}(S)=\sum_{A=\left\{S_{1}, \ldots, S_{n}\right\}: \cup S_{i}=S} \widetilde{\rho}_{\mathrm{f}}(A)
$$

This leads to

$$
\frac{Z_{W}^{-} \text {flat }}{Z_{W}^{+}\left(\Omega^{\prime}\right)}=e^{-\beta K A\left(\Omega^{\prime}\right)} \sum_{\substack{\left\{\gamma_{1}, \ldots, \gamma_{n}\right\} \text { comp } \\ \gamma_{i} \cap \Pi \neq \emptyset}} \prod_{i=1}^{n} z_{\mathrm{f}}^{-}\left(\gamma_{i}\right) \sum_{\substack{\left\{S_{1}, \ldots, S_{m}\right\}_{c o m p} \\ S_{j} \cap \Pi \neq \emptyset \text { orn } \cap S_{j} \nsim \gamma_{i}}} \prod_{j=1}^{m} \rho_{\mathrm{f}}\left(S_{j}\right)
$$


We call excitation a subset $\Gamma \subset\left\{\gamma_{1}, \ldots, \gamma_{n}\right\}_{\text {comp }} \cup\left\{S_{1}, \ldots, S_{m}\right\}_{\text {comp }}$ whose support supp $\Gamma=\left(\cup_{\gamma \in \Gamma} \gamma\right) \cup\left(\cup_{S \in \Gamma}\right)$ is connected and define the weight of an excitation $\Gamma$ by:

$$
\omega_{\mathrm{f}}(\Gamma)=\prod_{\gamma \in \Gamma} z_{\mathrm{f}}^{-}(\gamma) \prod_{S \in \Gamma} \rho_{\mathrm{f}}(S)
$$

Then

$$
\frac{Z_{W^{\text {flat }}}^{-}\left(\Omega^{\prime}\right)}{Z_{W^{\text {flat }}}^{+}\left(\Omega^{\prime}\right)}=e^{-\beta K A\left(\Omega^{\prime}\right)} \sum_{\substack{\left\{\Gamma_{1}, \ldots, \Gamma_{n}\right\}_{\operatorname{comp}} \\ \operatorname{supp} \Gamma_{i} \cap \Pi \neq \emptyset}} \prod_{i=1}^{n} \omega_{\mathrm{f}}\left(\Gamma_{i}\right)
$$

where the sum runs over compatible families $\left\{\Gamma_{1}, \ldots, \Gamma_{n}\right\}_{\text {comp }}$ of excitations.

For $J / \rho<K<J$ the weights satisfy the bound

$$
\begin{aligned}
& \left|z_{\mathrm{f}}^{+}(\gamma)\right| \leq e^{-\beta K|\gamma|} \\
& \left|z_{\mathrm{f}}^{-}(\gamma)\right| \leq e^{-\beta\left(\frac{J-K}{2}\right)|\gamma|}
\end{aligned}
$$

The truncated functional are then bounded as:

$$
\left|\Phi_{\mathrm{f}}^{+}(S)\right| \leq|S|\left(\kappa \nu e^{-\beta K}\right)^{|S|}
$$

Here the cluster constant may be computed as $\kappa=1+2(\sqrt{2}+1) e^{\frac{2}{1+\sqrt{2}}}$ and the entropy as $\nu=3^{2} 2^{8}$ (see [12]). The weights of aggregates may be controlled with the inequality $\left|e^{-\Phi_{\mathrm{f}}^{+}(S)}-1\right| \leq(e-1)\left|\Phi_{\mathrm{f}}^{+}(S)\right|$. This allows to show (see again [12]):

$$
\left|\rho_{\mathrm{f}}(S)\right| \leq\left|8 e(e-1) \kappa \nu^{2} e^{-\beta K}\right|^{|S|}
$$

provided $8 e(e-1) \kappa \nu^{2} e^{-\beta K} \leq 1$.

We will now exponentiate the R.H.S. of (5.15). To this ends we introduce multi-indexes $C$ defined on the set of excitations i.e. as functions from the set of excitations into the set of non negative integers. We let $\operatorname{supp} C=$ $\{\Gamma: C(\Gamma) \geq 1\}$ and let $\Psi_{\mathrm{f}}$ be the truncated functional associated to $\omega_{\mathrm{f}}$ :

$$
\Psi_{\mathrm{f}}(C)=\frac{a(C)}{\prod_{\gamma} C(\Gamma) !} \prod_{\Gamma} \omega_{\mathrm{f}}(\Gamma)^{C(\Gamma)}
$$

where $a(C)$ is defined as in (4.7) with a graph $G(C)$ whose vertices correspond to excitations $\Gamma \in \operatorname{supp} C$ and that are connected by an edge whenever the 
corresponding excitations are incompatible. We get as a result of cluster expansion

$$
\ln \frac{Z_{W_{\text {flat }}^{-}}^{-}\left(\Omega^{\prime}\right)}{Z_{W^{\text {flat }}}^{+}\left(\Omega^{\prime}\right)}+\beta K A\left(\Omega^{\prime}\right)=\sum_{C \in \chi_{\Pi}\left(\Omega^{\prime}\right)} \Psi_{\mathrm{f}}(C)
$$

Using that the weights are now completely invariant with respect to horizontal translations, we have taking $\Omega^{\prime}=\Lambda(L) \cap \mathbf{L}$

$$
(\Delta \tau)^{\mathrm{flat}}-K=\lim _{L \rightarrow \infty} \frac{1}{\beta(2 L+1)^{2}} \sum_{C \in \chi_{\Pi}\left(\Omega^{\prime}\right)} \Psi_{\mathrm{f}}(C)=\sum_{\substack{C \in \chi_{\Pi}(\mathbb{I}) \\ p \in C}} \frac{\Psi_{\mathrm{f}}(C)}{|C \cap \Pi|} \equiv \mathcal{F}_{\mathrm{f}}
$$

Here the last sum is over multi-indexes whose support contains a given plaquette of the plane $\Pi$. This series converges provided one can find a positive function such that

$$
\omega(\Gamma) \exp \left\{-\sum_{\Gamma^{\prime} \nsim \Gamma} \mu\left(\Gamma^{\prime}\right)\right\} \leq \mu(\Gamma)
$$

This condition is fulfilled whenever

$$
2 \nu \kappa \max \left(e^{-\beta\left(\frac{J-K}{2}\right)}, 8 e(e-1) \kappa \nu^{2} e^{-\beta K}\right) \leq 1
$$

To see it, we put $\mu(\Gamma)=(2 \nu a)^{-|\Gamma|}$ where $|\Gamma|$ is the number of plaquettes of supp $\Gamma$, getting $\sum_{\Gamma^{\prime} \nsim \Gamma} \mu\left(\Gamma^{\prime}\right) \leq \frac{2^{2}}{a-1}|\Gamma|$. We then choose for $a$ the value $1+2(1+\sqrt{2})$ that minimizes the function $a e^{\frac{2^{2}}{a-1}}$.

\subsection{The surface tension $\tau_{+-}$}

We now turn to the surface tension $\tau_{+-}$. An important property of this surface tension is that it can be defined as an appropriated limit of (2.4) with many different boxes $\Lambda$ [4]. Indeed, one can take instead of $\Lambda(L)$, the set

$$
\left\{x \in \mathbb{Z}^{3}:\left|x_{1}\right| \leq L,\left|x_{2}\right| \leq L, h(L)<x_{3} \leq g(L)\right\}
$$

provided the height functions $-h$ and $g$ goes to infinity in the limit when $\mathrm{L}$ tends to infinity. Here we shall consider the box

$$
\Omega_{c, b}=\left\{x \in \mathbb{Z}^{3}: 0 \leq x_{1} \leq c, 0 \leq x_{2} \leq c,-b \leq x_{1} \leq L\right\}
$$


and let $Z^{+}\left(\Omega_{c, b}\right)$ be the partition function of the Ising model in the box $\Omega_{c, b}$ with + boundary condition and $Z^{+-}\left(\Omega_{c, b}\right)$ be the partition function with + boundary condition below the plane $\Pi$ and - boundary condition above this plane. Then

$$
\beta \tau_{+-}=-\lim _{L \rightarrow \infty} \frac{1}{c^{2}} \ln \frac{Z^{+-}\left(\Omega_{c, b}\right)}{Z^{+}\left(\Omega_{c, b}\right)}
$$

Instead of $\tau_{+-}$we shall study an auxiliary differential tension $(\Delta \tau)_{\text {aux }}$ that will eventually coincide with $\tau_{+-}$. Consider the box $\Omega_{c, b}$ (associated to a single pore), and let $Z_{W \text { pore }}^{+}\left(\Omega_{c, b}\right)$ and $Z_{W \text { pore }}^{-}\left(\Omega_{c, b}\right)$ be the partitions functions corresponding to the Hamiltonian (2.1) in the box $\Omega_{c, b}$ with+ and boundary conditions respectively. This means that the partition function $Z_{W^{\text {pore }}}^{+}\left(\Omega_{c, b}\right)$ (resp. $Z_{W^{\text {pore }}}^{-}\left(\Omega_{c, b}\right)$ ) differs from the partition function $Z^{+}\left(\Omega_{c, b}\right)$ (resp. $Z^{+-}\left(\Omega_{c, b}\right)$ ) only by the fact that the coupling between bonds $x y$ $x \in \Omega_{c, b} y \notin \Omega_{c, b}$ below the plane $\Pi$ is $K$ instead of $J$. We define

$$
\beta(\Delta \tau)_{\mathrm{aux}}=-\lim _{L \rightarrow \infty} \frac{1}{c^{2}} \ln \frac{Z_{W^{\mathrm{pore}}}^{-}\left(\Omega_{c, b}\right)}{Z_{W^{\mathrm{pore}}}^{+}\left(\Omega_{c, b}\right)}
$$

For the partition function $Z_{W \text { pore }}^{-}\left(\Omega_{c, b}\right)$, we define contours as connected component of the set of all plaquettes separating nearest neighbour sites $x, y$ for which $\sigma_{x} \neq \sigma_{y}$ if the bond $x y$ does not cross the plane $\Pi$ or nearest neighbour sites $x, y$ for which $\sigma_{x}=\sigma_{y}$ if the bond $x y$ crosses the plane $\Pi$. We introduce the weights

$$
z_{\mathrm{pr}}^{-}(\gamma)=e^{-\beta\left(J\left|\gamma_{\mathrm{bk}}\right|-J\left|\gamma_{0}\right|+K\left|\gamma_{\mathrm{pr}}\right|\right.}
$$

Here $\gamma_{0}=\gamma \cap \Pi$ is the set of dual plaquettes of bond crossing the plane $\Pi$, $\gamma_{\mathrm{pr}}$ is the set of dual plaquettes of bonds $x y, x \in \Omega_{c, b} y \notin \Omega_{c, b}$ below the plane $\Pi$ and $\gamma_{\mathrm{bk}}=\gamma \backslash\left(\gamma_{0} \cap \gamma_{\mathrm{pr}}\right)$. We will say that the contour $\gamma$ touches the wall if $\gamma_{\text {pr }}$ is not empty. Then,

$$
Z_{W^{\text {pore }}}^{-}\left(\Omega_{c, b}\right)=e^{-\beta J A\left(\Omega_{c, b}\right)} \sum_{\left\{\gamma_{1}, \ldots, \gamma_{n}\right\}_{\text {comp }}} \prod_{i=1}^{n} z_{\mathrm{pr}}^{-}\left(\gamma_{i}\right)
$$

where $A\left(\Omega_{c, b}\right)$ is the number of bonds $x y$ of $\Omega_{c, b}$ that crosses the plane $\Pi$ (and $\left\{\gamma_{1}, \ldots, \gamma_{n}\right\}_{\text {comp }}$ is a collection of compatible contours in $\Omega_{c, b}$ ). 
For the partition function $Z_{W}^{+}$pore $\left(\Omega_{c, b}\right)$ we use the standard representation of contours and define the weights

$$
z_{\mathrm{pr}}^{+}(\gamma)=e^{-\beta\left(J\left|\gamma_{\mathrm{bk}}\right|+J\left|\gamma_{0}\right|+K\left|\gamma_{\mathrm{pr}}\right|\right.}
$$

getting

$$
\begin{aligned}
Z_{W^{\text {pore }}}^{+}\left(\Omega_{c, b}\right) & =\sum_{\left\{\gamma_{1}, \ldots, \gamma_{n}\right\}_{\text {comp }}} \prod_{i=1}^{n} z_{\mathrm{pr}}^{+}\left(\gamma_{i}\right)=\exp \left(\sum_{X \in \chi\left(\Omega_{c, b}\right)} \widetilde{\Phi}_{\mathrm{pr}}^{+}(X)\right) \\
& =\exp \left(\sum_{S \in \chi\left(\Omega_{c, b}\right)} \Phi_{\mathrm{pr}}^{+}(S)\right)
\end{aligned}
$$

where $\widetilde{\Phi}_{\mathrm{pr}}^{+}$is the truncated functional associated to $z_{\mathrm{pr}}^{+}$, and as above we have summed over all multi-indexes with same support:

$$
\Phi_{\mathrm{pr}}^{+}(S)=\sum_{X: \operatorname{supp} X=S} \widetilde{\Phi}_{\mathrm{pr}}^{+}(X)
$$

Since by definitions the weights of contours are the same for contours not touching the plane $\Pi$, we have

$$
Z_{W^{\text {pore }}}^{-}\left(\Omega_{c, b}\right)=e^{-\beta J A\left(\Omega_{c, b}\right)} \sum_{\substack{\left\{\gamma_{1}, \ldots, \gamma_{n}\right\}_{c o m p} \\ \gamma_{i} \cap \Pi \neq \emptyset}} \prod_{i=1}^{n} z_{\mathrm{pr}}^{-}\left(\gamma_{i}\right) \sum_{\substack{\left.\left\{\gamma_{1}^{\prime}, \ldots, \gamma_{m}^{\prime}\right\}\right\}_{c o m p} \\ \gamma_{j}^{\prime} \cap \Pi=\emptyset, \gamma_{j}^{\prime} \sim \gamma_{i}}} \prod_{i=1}^{m} z_{\mathrm{pr}}^{+}\left(\gamma_{j}^{\prime}\right)
$$

Here the first sum is over (compatible) families of contours touching the plane $\Pi$ and the second ones is over (compatible) families of contours not touching the plane $\Pi$ and compatible with the first family.

By taking into account (5.27), one gets

$$
\begin{aligned}
\frac{Z_{W \text { pore }}^{-}\left(\Omega_{c, b}\right)}{Z_{W \text { pore }}^{+}\left(\Omega_{c, b}\right)} & =e^{-\beta J A\left(\Omega_{c, b}\right)} \sum_{\substack{\left\{\gamma_{1}, \ldots, \gamma_{n}\right\}_{c o m p} \\
\gamma_{i} \cap \Pi \neq \emptyset}} \prod_{i=1}^{n} z_{\mathrm{pr}}^{-}\left(\gamma_{i}\right) \exp \left(-\sum_{\substack{S: S \in \chi_{\Pi}\left(\Omega_{c, b}\right) \\
\text { ors } S \gamma_{i}}} \Phi_{\mathrm{pr}}^{+}(S)\right) \\
& =e^{-\beta J A\left(\Omega_{c, b}\right)} \sum_{\substack{\left\{\gamma_{1}, \ldots, \gamma_{n}\right\}_{c o m p} \\
\gamma_{i} \cap \Pi \neq \emptyset}} \prod_{i=1}^{n} z_{\mathrm{pr}}^{-}\left(\gamma_{i}\right) \prod_{\substack{S: S \in \chi_{\Pi}\left(\Omega_{c, b}\right) \\
\text { or } S \nsim \gamma_{i}}} e^{-\Phi_{\mathrm{pr}}^{+}(S)}
\end{aligned}
$$


As above, to expand the last product we introduce aggregates $A$ as families of clusters whose support is connected and define the weights $\widetilde{\rho}_{\mathrm{pr}}(A)=$ $\prod_{S \in A} e^{-\Phi_{\mathrm{pr}}^{+}(S)}-1$ to get

$$
\frac{Z_{W^{\text {pore }}}^{-}\left(\Omega_{c, b}\right)}{Z_{W^{\text {pore }}}^{+}\left(\Omega_{c, b}\right)}=e^{-\beta J A\left(\Omega_{c, b}\right)} \sum_{\substack{\left\{\gamma_{1}, \ldots, \gamma_{n}\right\}_{c o m p} \\ \gamma_{i} \cap \Pi \neq \emptyset}} \prod_{i=1}^{n} z_{\text {pr }}^{-}\left(\gamma_{i}\right) \sum_{\substack{\left\{A_{1}, \ldots, A_{m}\right\}_{\text {comp }} \\ A_{j} \cap \Pi \neq \emptyset \text { or } \cap A_{j} \nsim \gamma_{i}}} \prod_{j=1}^{m} \widetilde{\rho}_{\mathrm{pr}}\left(A_{j}\right)
$$

Here again, it is convenient to sum over all aggregates with the same support. We thus define the weights

$$
\rho_{\mathrm{pr}}(S)=\sum_{A=\left\{S_{1}, \ldots, S_{n}\right\}: \cup S_{i}=S} \widetilde{\rho}_{\mathrm{pr}}(A)
$$

getting

$$
\frac{Z_{W \text { pore }}^{-}\left(\Omega_{c, b}\right)}{Z_{W^{\text {pore }}}^{+}\left(\Omega_{c, b}\right)}=e^{-\beta J A\left(\Omega_{c, b}\right)} \sum_{\substack{\left\{\gamma_{1}, \ldots, \gamma_{n}\right\}_{\text {comp }} \\ \gamma_{i} \cap \Pi \neq \emptyset}} \prod_{i=1}^{n} z_{\mathrm{pr}}^{-}\left(\gamma_{i}\right) \sum_{\substack{\left\{S_{1}, \ldots, S_{m}\right\}_{\text {comp }} \\ S_{j} \cap \Pi \neq \neq \emptyset \text { ornS } S_{j} \nsim \gamma_{i}}} \prod_{j=1}^{m} \rho_{\mathrm{pr}}\left(S_{j}\right)
$$

Notice that the weight $z_{\text {pr }}^{-}(\gamma)$ do not always decrease with the area of contours. To control the ratio of the two partition functions above in terms of convergent cluster expansion, we have to define the right excitations. To this end, we first split the set $\left\{\gamma_{1}, \ldots, \gamma_{n}\right\}_{\text {comp }} \cup\left\{S_{1}, \ldots, S_{m}\right\}_{\text {comp }}$ in connected components. The components whose support touches the wall $W^{\text {pore }}$ are called wall excitations and denoted $\Gamma^{\text {wall }}$. We use $B_{\text {pr }}$ to denote the subset composed of wall excitations and $B_{\mathrm{bk}}$ to denote its complement. For the wall excitations, we define the weights

$$
\omega_{\mathrm{pr}}\left(\Gamma^{\text {wall }}\right)=\prod_{\gamma \in \Gamma_{\text {wall }}} z_{\mathrm{pr}}^{-}(\gamma) \prod_{S \in \Gamma \text { wall }} \rho_{\mathrm{pr}}(S)
$$

Note that $\left|z_{\mathrm{f}}^{+}(\gamma)\right| \leq e^{-\beta K|\gamma|}$ and therefore $\rho_{\mathrm{pr}}(S)$ satisfy the bound (5.18). On the other hand, for the wall excitations one has

$$
\left|z_{\mathrm{f}}^{-}(\gamma)\right| \leq e^{-\beta\left(\frac{\rho|K|-J}{1+\rho}\right)|\gamma|}
$$

for any $\gamma \in \Gamma^{\text {wall }}$. This implies that the weights (5.30) have good decaying properties for large $\beta$. 
For the remaining part $B_{\mathrm{bk}}$, this is not the case and we have to introduce the excitations differently. In the situation under consideration, they can be defined following the Dobrushin's analysis given in [14] (see also [5, 6, 21]). Namely, for any component $B \in B_{\mathrm{bk}}$ and any contour $\gamma \in B$ touching the plane $\Pi$, we will divide the set of plaquettes of $\gamma$ in two sets. An horizontal plaquette $p \in \gamma$ is called correct (or ceiling face in the terminology of [14]) if it lies on the plane $\Pi$ or if the vertical lines that crosses it in its middle crosses only two horizontal plaquettes of $B_{\mathrm{bk}}$. All the other plaquettes of $\gamma$ are called incorrect (or wall faces in the terminology of [14]). We use $I\left(B_{\mathrm{bk}}\right)$ to denote the set of incorrect plaquettes of $B_{\mathrm{bk}}$. Then the union of $I\left(B_{\mathrm{bk}}\right)$ with the set of clusters $S \in B_{\mathrm{bk}}$ splits into connected components $\Gamma^{\mathrm{el}}=\left\{p_{1}, . ., p_{n} ; S_{1}, \ldots, S_{m}\right\}$ called elementary excitations (or walls in the terminology of [14]). A set $B_{\mathrm{bk}}=\left\{\gamma_{1}, \ldots, \gamma_{n}\right\}$ such that $\gamma_{i} \cap \Pi \neq \emptyset$ is in one-to-one correspondence with a set of elementary excitations. An elementary excitation $\Gamma^{\mathrm{el}}=\left\{p_{1}, . ., p_{n} ; S_{1}, \ldots, S_{m}\right\}$ is said in the standard position if there exists a contour $\gamma$ such that $\left\{p_{1}, . ., p_{n}\right\}$ is the only elementary excitation corresponding to $\gamma$.

Let $T_{h}$ denotes the vertical shift by a height $h: T_{h}(x)=\left(x_{1}, x_{2}, x_{3}+h\right)$, $T_{h}(A)=\left\{x: T_{h}^{-1}(x) \in A\right\}$. Then for any elementary excitation, there is only one shifted excitation $\Gamma^{\mathrm{sh}}=T_{h}\left(\Gamma^{\mathrm{el}}\right)$ which is in the standard position (see [14] or Lemma 2.2 in [18]). We define the weights of any shifted or elementary excitation by

$$
\omega_{\mathrm{pr}}\left(\Gamma^{\mathrm{sh}}\right)=\omega_{\mathrm{pr}}\left(\left\{p_{1}, . ., p_{n} ; S_{1}, \ldots, S_{m}\right\}\right)=e^{-\beta J n} \prod_{j=1}^{m} \rho_{\mathrm{pr}}\left(S_{j}\right)
$$

With these definitions, we get from (5.29):

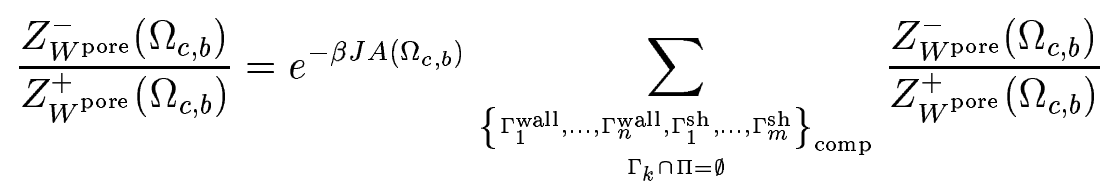

where $\left\{\Gamma_{1}^{\text {wall }}, \ldots, \Gamma_{n}^{\text {wall }}, \Gamma_{1}^{\text {sh }}, \ldots, \Gamma_{m}^{\text {sh }}\right\}_{\text {comp }}$ are families of (compatible) walls or shifted excitations whose support touches the plane $\Pi$. We introduce as before the multi-indexes $C$ as non compatible families of excitations and let $\Psi_{\mathrm{pr}}$ be the corresponding truncated functional to get

$$
\ln \frac{Z_{W^{\text {pore }}}^{-}\left(\Omega_{c, b}\right)}{Z_{W^{\text {pore }}}^{+}\left(\Omega_{c, b}\right)}+\beta J A\left(\Omega_{c, b}\right)=\sum_{C \in \chi_{\Pi}\left(\Omega_{c, b}\right)} \Psi_{\mathrm{pr}}(C)
$$


Here, the convergence condition reads

$$
2 \nu \kappa \max \left(e^{-\beta\left(\frac{\rho|K|-J}{1+\rho}\right)}, 8 e(e-1) \kappa \nu^{2} e^{-\beta K}\right) \leq 1
$$

By definition (5.23) of the auxiliary tension $(\Delta \tau)_{\text {aux }}$, this relation gives

$$
\beta(\Delta \tau)_{\mathrm{aux}}-\beta J=-\lim _{L \rightarrow \infty} \frac{1}{c^{2}} \sum_{\substack{C \in \chi_{\Pi}\left(\Omega_{c, b}\right) \\ \text { supp } C \cap W^{\mathrm{pore}}=\emptyset}} \Psi_{\mathrm{pr}}(C)-\lim _{L \rightarrow \infty} \frac{1}{c^{2}} \sum_{\substack{C \in \chi_{\Pi}\left(\Omega_{c, b}\right) \\ \text { supp } C \cap W^{\mathrm{pore}} \neq \emptyset}} \Psi_{\mathrm{pr}}(C)
$$

where supp $C=\cup_{\Gamma \in C} \operatorname{supp} \Gamma$. The second limit actually goes to zero. This may be seen by realizing that the sum over multi-indexes whose support touches both the plane $\Pi$ and $W^{\text {pore }}$ is composed of a term proportional to the perimeter of the square $c^{2}$ (for multi-indexes touching the vertical faces of $W^{\text {pore }}$ ) plus a term proportional to $c^{2}$ times an exponential small correction proportional to $b$ (for multi-indexes touching the horizontal face of $W^{\text {pore }}$. The first limit gives actually the free energy of excitations of the surface tension $\tau_{+-}$so that

$$
(\Delta \tau)_{\mathrm{aux}}=\tau_{+-}
$$

\subsection{The differential tension $\Delta \tau$}

We finally turn to the differential wall tension of the rough wall. For the partition function $Z_{W}^{-}(\Omega)$, we have again to be careful with the definition of contours. For configurations $\sigma$ (such that $\sigma_{x}=+1$ for $x \in W$ and $\sigma_{x}=-1$ for $x \in \Omega^{c} \backslash W$ ), we introduce now contours as connected component of the set $B^{0}(\sigma)$ of all plaquettes separating nearest neighbour sites $x, y$ for which $\sigma_{x} \neq \sigma_{y}$ if the bond $x y$ does not cross the plane $\Pi$ or nearest neighbour sites $x, y$ for which $\sigma_{x}=\sigma_{y}$ if the bond $x y$ crosses the plane $\Pi$. We define the set $(\partial W)_{\mathrm{f}}=\Pi \cap \partial W$, to be the part of the boundary of the wall intersecting the plane $\Pi$, the set $(\partial W)_{\mathrm{pr}}=\partial W \backslash(\partial W)_{\mathrm{f}}$ to be its complement and the set $\Pi_{0}=\Pi \backslash(\partial W)_{\mathrm{f}}$ to be the complement of $(\partial W)_{\mathrm{f}}$.

From the definition of $B^{0}(\sigma)$ (defined as the boundary of regions where the configuration differs from the ground state $\sigma_{0}^{-}$), it follows that the configuration $\sigma_{\gamma}$ associated to the unique contour $\gamma$ satisfy:

$$
H_{\Omega}^{-}\left(\sigma_{\gamma}\right)-H_{\Omega}^{-}\left(\sigma_{0}^{-}\right)=J\left|\gamma_{\mathrm{bk}}\right|+K\left|\gamma_{\mathrm{pr}}\right|-K\left|\gamma_{\mathrm{f}}\right|-J\left|\gamma_{0}\right|
$$

where $\gamma_{\mathrm{pr}}=\gamma \cap(\partial W)_{\mathrm{pr}}, \gamma_{\mathrm{f}}=\gamma \cap(\partial W)_{\mathrm{f}}$ is the part of the contour that intersect $(\partial W)_{\mathrm{pr}}$, respectively $(\partial W)_{\mathrm{f}}, \gamma_{0}=\gamma \cap \Pi_{0}$ is the part of the contour 
that intersect $\Pi_{0}$, and $\gamma_{\mathrm{bk}}=\gamma \backslash\left(\gamma_{\mathrm{pr}} \cup \gamma_{\mathrm{fl}} \cup \gamma_{0}\right)$ is the complement of these three sets. Introducing now the weight $z^{-}(\gamma)$ as

$$
z^{-}(\gamma)=e^{-\beta\left(J\left|\gamma_{\mathrm{bk}}\right|+K\left|\gamma_{\mathrm{pr}}\right|-K\left|\gamma_{\mathbf{f}}\right|-J\left|\gamma_{0}\right|\right)}
$$

we get

$$
Z_{W}^{-}(\Omega)=e^{-\beta\left[K A_{\mathrm{f}}(\Omega)+J A_{0}(\Omega)\right]} \sum_{\left\{\gamma_{1}, \ldots, \gamma_{n}\right\}_{\mathrm{comp}}} \prod_{i=1}^{n} z^{-}\left(\gamma_{i}\right)
$$

where $A_{\mathrm{f}}(\Omega)$, is the number of bonds $x y, x \in \Omega, y \in W$ that crosses $(\partial W)_{\mathrm{f}}$ and $A_{0}(\Omega)$ is the number of bonds $x y, x \in \Omega, y \in \Omega$ that crosses $\Pi_{0}$.

For $Z_{W}^{+}(\Omega)$ we keep the standard definitions of contours, so that introducing the weight factors

$$
z^{+}(\gamma)=e^{-\beta\left(J\left|\gamma_{\mathrm{bk}}\right|+K\left|\gamma_{\mathrm{pr}}\right|+K\left|\gamma_{\mathrm{f}}\right|+J\left|\gamma_{0}\right|\right)}
$$

we get

$$
\begin{aligned}
Z_{W}^{+}(\Omega) & =\sum_{\left\{\gamma_{1}, \ldots, \gamma_{n}\right\}_{\mathrm{comp}}} \prod_{i=1}^{n} z^{+}\left(\gamma_{i}\right)=\exp \left(\sum_{X \in \chi(\Omega)} \widetilde{\Phi}^{+}(X)\right) \\
& =\exp \left(\sum_{S \in \chi(\Omega)} \Phi^{+}(S)\right)
\end{aligned}
$$

where $\widetilde{\Phi}^{+}$is the truncated functional associated to $z_{\mathrm{pr}}^{+}$, and as above we summed over all multi-indexes with same support:

$$
\Phi^{+}(S)=\sum_{X: \operatorname{supp} X=S} \widetilde{\Phi}^{+}(X)
$$

Note that the weights $z^{+}(\gamma)$ are bounded as

$$
\left|z^{+}(\gamma)\right| \leq e^{-\beta K|\gamma|}
$$

Since by definitions the weights of the contours not touching the plane $\Pi$ are exactly the same for + or - b.c., we have

$$
Z_{W}^{-}(\Omega)=e^{-\beta\left[K A_{\mathrm{f}}(\Omega)+J A_{0}(\Omega)\right]} \sum_{\substack{\left\{\gamma_{1}, \ldots, \gamma_{n}\right\} \text { comp } \\ \gamma_{i} \cap \Pi \neq \emptyset}} \prod_{i=1}^{n} z^{-}\left(\gamma_{i}\right) \sum_{\substack{\left\{\gamma_{1}^{\prime}, \ldots, \gamma_{m}^{\prime}\right\} \text { comp } \\ \gamma_{j}^{\prime} \cap \Pi=\emptyset, \gamma_{j}^{\prime} \sim \gamma_{i}}} \prod_{i=1}^{m} z^{+}\left(\gamma_{j}^{\prime}\right)
$$


which gives by taking into account (5.40)

$$
\begin{aligned}
\frac{Z_{W}^{-}(\Omega)}{Z_{W}^{+}(\Omega)} & =e^{-\beta\left[K A_{\mathrm{f}}(\Omega)+J A_{0}(\Omega)\right]} \sum_{\substack{\left\{\gamma_{1}, \ldots, \gamma_{n}\right\}_{\text {comp }} \\
\gamma_{i} \cap \Pi \neq \emptyset}} \prod_{i=1}^{n} z^{-}\left(\gamma_{i}\right) \exp \left(-\sum_{\substack{S: S \in \chi_{\Pi}(\Omega) \\
\text { ors } S \nsim \gamma_{i}}} \Phi^{+}(S)\right) \\
& =e^{-\beta\left[K A_{\mathrm{f}}(\Omega)+J A_{0}(\Omega)\right]} \sum_{\substack{\left\{\gamma_{1}, \ldots, \gamma_{n}\right\} \text { comp } \\
\gamma_{i} \cap \Pi \neq \emptyset}} \prod_{i=1}^{n} z^{-}\left(\gamma_{i}\right) \prod_{\substack{S: S \in \chi_{\Pi}(\Omega) \\
\text { or } S \nsim \gamma_{i}}} e^{-\Phi^{+}(S)}
\end{aligned}
$$

To expand the last product we introduce again aggregates $A$ as families of clusters $S$ whose support is connected and define the weights $\widetilde{\rho}(A)=$ $\prod_{S \in S} e^{-\Phi^{+}(A)}-1$ to get

$$
\frac{Z_{W}^{-}(\Omega)}{Z_{W}^{+}(\Omega)}=e^{-\beta\left[K A_{\mathrm{f}}(\Omega)+J A_{0}(\Omega)\right]} \sum_{\substack{\left\{\gamma_{1}, \ldots, \gamma_{n}\right\}_{c o m p} \\ \gamma_{i} \cap \Pi \neq \emptyset}} \prod_{i=1}^{n} z^{-}\left(\gamma_{i}\right) \sum_{\substack{\left\{A_{1}, \ldots, A_{m}\right\}_{\text {comp }} \\ A_{j} \cap \Pi \neq \emptyset \text { or } \cap A_{j} \nsim \gamma_{i}}} \prod_{j=1}^{m} \widetilde{\rho}\left(A_{j}\right)
$$

Again, we sum over all aggregates with the same support by defining the weights

$$
\rho(S)=\sum_{A=\left\{S_{1}, \ldots, S_{n}\right\}: \cup S_{i}=A} \widetilde{\rho}(A)
$$

to get

$$
\frac{Z_{W}^{-}(\Omega)}{Z_{W}^{+}(\Omega)}=e^{-\beta\left[K A_{\mathrm{f}}(\Omega)+J A_{0}(\Omega)\right]} \sum_{\substack{\left\{\gamma_{1}, \ldots, \gamma_{n}\right\}_{c o m p} \\ \gamma_{i} \cap \Pi \neq \varnothing \emptyset}} \prod_{i=1}^{n} z^{-}\left(\gamma_{i}\right) \sum_{\substack{\left\{S_{1}, \ldots, S_{m}\right\}_{\text {comp }} \\ S_{j} \cap \Pi \neq \emptyset \text { or or } S_{j} \nsim \gamma_{i}}} \prod_{j=1}^{m} \rho\left(S_{j}\right)
$$

As in the previous section the weights $z^{-}(\gamma)$ have good decaying properties only for contours touching the wall. To control the ratio, we proceed as for the study of the surface tension $\tau_{+-}$. Namely, we first split the set $\left\{\gamma_{1}, \ldots, \gamma_{n}\right\}_{\text {comp }} \cup\left\{S_{1}, \ldots, S_{m}\right\}_{\text {comp }}$ in connected components. The components whose support touches the wall $W$ are called wall excitations and denoted $\Gamma^{\text {wall }}$. We use $B_{W}$ to denote the subset of $\left\{\gamma_{1}, \ldots, \gamma_{n}\right\}_{\text {comp }} \cup\left\{S_{1}, \ldots, S_{m}\right\}_{\text {comp }}$ composed of wall excitations and $B_{\mathrm{bk}}$ to denote its complement. For the wall excitations, we define the weights

$$
\omega\left(\Gamma^{\text {wall }}\right)=\prod_{\gamma \in \Gamma^{\text {wall }}} z^{-}(\gamma) \prod_{S \in \Gamma_{\text {wall }}} \rho(S)
$$


Since $\left|z_{\mathrm{f}}^{+}(\gamma)\right| \leq e^{-\beta K|\gamma|}, \rho_{\mathrm{pr}}(S)$ satisfy the bound (5.18). On the other hand, for these wall excitations one has

$$
\left|z_{\mathrm{f}}^{-}(\gamma)\right| \leq \min \left(e^{-\beta\left(\frac{J-K}{2}\right)|\gamma|}, e^{-\beta\left(\frac{\rho|K|-J}{1+\rho}\right)|\gamma|}\right)
$$

for any $\gamma \in \Gamma^{\text {wall }}$. This implies that the weights (5.30) have good decaying properties for large $\beta$.

For the remaining part $B_{\mathrm{bk}}$ this is not the case and we have to introduce the excitations differently. We shall define them as in the study of the auxiliary tension $(\Delta \tau)_{\mathrm{aux}}$. Namely, for any $B \in B_{\mathrm{bk}}$ and any contour $\gamma \in B$ touching the plane $\Pi$, we will divide the set of plaquettes of $\gamma$ in two sets. A plaquette $p \in \gamma$ is called correct if it lies on the plane $\Pi$ or if the vertical lines that crosses it in its middle crosses only two horizontal plaquettes of $B_{\mathrm{bk}}$. All the other plaquettes of $\gamma$ are called incorrect: in particular, all the vertical plaquettes are incorrect ones. We use $I\left(B_{\mathrm{bk}}\right)$ to denote the set of incorrect plaquettes of $B_{\mathrm{bk}}$. Then the union of $I\left(B_{\mathrm{bk}}\right)$ with the set clusters $S \in B_{\mathrm{bk}}$ split into connected components $\left\{p_{1}, . ., p_{n} ; S_{1}, \ldots, S_{m}\right\}$ called elementary excitations. A set $B_{\mathrm{bk}}=\left\{\gamma_{1}, \ldots, \gamma_{n}\right\}$ such that $\gamma_{i} \cap \Pi \neq \emptyset$ is in one-to-one correspondence with a set of elementary excitations. An elementary excitation $\Gamma^{\mathrm{el}}=\left\{p_{1}, . ., p_{n} ; S_{1}, \ldots, S_{m}\right\}$ is said in the standard position if there exists a contour $\gamma$ such that $\left\{p_{1}, . ., p_{n}\right\}$ is the only elementary excitation corresponding to $\gamma$.

Let $T_{h}$ denotes the vertical shift by a height $h: T_{h}(x)=\left(x_{1}, x_{2}, x_{3}+h\right)$, $T_{h}(A)=\left\{x: T_{h}^{-1}(x) \in A\right\}$. Then for any elementary excitation, there is only one shifted excitation $\Gamma^{\mathrm{sh}}=T_{h}\left(\Gamma^{\mathrm{el}}\right)$ which is in the standard position. We define the weights of any shifted or elementary excitation by

$$
\omega\left(\Gamma^{\mathrm{sh}}\right)=\omega\left(\left\{p_{1}, . ., p_{n} ; S_{1}, \ldots, S_{m}\right\}\right)=e^{-\beta J n} \prod_{j=1}^{m} \rho\left(S_{j}\right)
$$

With these definitions, we get from (5.45):

$$
\frac{Z_{W}^{-}(\Omega)}{Z_{W}^{+}(\Omega)}=e^{-\beta\left[K A_{\mathrm{f}}(\Omega)+J A_{0}(\Omega)\right]} \sum_{\substack{\left\{\Gamma_{1}^{\mathrm{wall}}, \ldots, \Gamma_{n}^{\mathrm{wall}}, \Gamma_{1}^{\mathrm{sh}}, \ldots, \Gamma_{m}^{\mathrm{sh}}\right\}_{\text {comp }} \\ \Gamma_{k} \cap \Pi=\emptyset}} \prod_{i=1}^{n} \omega\left(\Gamma_{i}^{\mathrm{wall}}\right) \prod_{j=1}^{m} \omega\left(\Gamma_{i}^{\mathrm{sh}}\right)
$$

where $\left\{\Gamma_{1}^{\text {wall }}, \ldots, \Gamma_{n}^{\text {wall }}, \Gamma_{1}^{\text {sh }}, \ldots, \Gamma_{m}^{\text {sh }}\right\}_{\text {comp }}$ are families of (compatible) wall or shifted excitations whose support touches the plane $\Pi$. We introduce as 
before the multi-indexes $C$ as non compatible families of excitations and let $\Psi$ be the corresponding truncated functional associated to $\omega$ to get

$$
\ln \frac{Z_{W}^{-}(\Omega)}{Z_{W}^{+}(\Omega)}+\beta\left[K A_{\mathrm{f}}(\Omega)+J A_{0}(\Omega)\right]=\sum_{C \in \chi_{\Pi}(\Omega)} \Psi(C)
$$

Using the fact that $\Psi(C)$ are invariant under horizontal translation by multiples of the periodicity constant $a$, one gets,

$$
\begin{aligned}
\Delta \tau-\left(1-c^{\prime}\right) K-c^{\prime} J & =\lim _{L \rightarrow \infty} \frac{1}{\beta(2 L+1)^{2}} \sum_{C \in \chi_{\Pi}(\Omega)} \Psi(C) \\
& =\lim _{a \rightarrow \infty} \frac{1}{\beta a^{2}} \sum_{C \in \chi_{\Pi}\left(\Omega_{a}\right)} \Psi(C)
\end{aligned}
$$

where $\Omega_{a}=V \cap \Lambda_{a}$, with

$$
\Lambda_{a}=\left\{x \in \mathbb{Z}^{3}: 0 \leq x_{1} \leq a, 0 \leq x_{2} \leq a,\left|x_{3}\right| \leq a\right\}
$$

To fulfill the convergence conditions we need to take

$$
2 \nu \kappa \max \left(e^{-\beta\left(\frac{J-K}{2}\right)}, e^{-\beta\left(\frac{\rho K-J}{1+\rho}\right)}, 8 e(e-1) \kappa \nu^{2} e^{-\beta K}\right) \leq 1
$$

Our last step is to compare the R.H.S. of (5.49) with (5.21) and (5.34). We shall take the box $\Omega^{\prime}$ to be the complement of $\Omega_{c, b}$ in $\Omega_{a}$

$$
\Omega^{\prime}=\Omega_{a} \backslash \Omega_{c, b}
$$

Then we split the sum over multi-indexes $C \in \chi_{\Pi}\left(\Omega_{a}\right)$ in three terms $S_{\mathrm{f}}(a)$, $S_{\mathrm{pr}}(a)$, and $R(a)$. The first sum $S_{\mathrm{f}}(a)$ is over multi-indexes whose support lies inside $\Omega^{\prime}$. Notice that for the multi-indexes $C$ involved in this sum, one has $\Psi(C)=\Psi_{\mathrm{f}}(C)$ and thus

$$
\lim _{a \rightarrow \infty} \frac{S_{\mathrm{f}}(a)}{\beta a^{2}}=\frac{a^{2}-c^{2}}{a^{2}} \mathcal{F}_{\mathrm{f}}=\left(1-c^{\prime}\right) \mathcal{F}_{\mathrm{f}}
$$

The second sum $S_{\mathrm{pr}}(a)$ is over multi-indexes whose support lies inside $\Omega_{c, b}$. For the multi-indexes $C$ involved in this sum, one has $\Psi(C)=\Psi_{\mathrm{pr}}(C)$ and thus

$$
\lim _{a \rightarrow \infty} \frac{S_{\mathrm{f}}(a)}{\beta a^{2}}=\frac{c^{2}}{a^{2}} \mathcal{F}_{\mathrm{pr}}=c^{\prime} \mathcal{F}_{\mathrm{pr}}
$$


Finally the reminder $R(a)$ contains the multi-indexes whose support intersects both $\Omega^{\prime}$ and $\Omega_{c, b}$. This term is thus bounded by a constant times the length of the separation line between and $\Pi_{0}$. Therefore the limit $R(a) / a^{2}$ goes to zero as $a \rightarrow \infty$ and we get

$$
\Delta \tau-\left(1-c^{\prime}\right) K-c^{\prime} J=\left(1-c^{\prime}\right) \mathcal{F}_{\mathrm{f}}+c^{\prime} \mathcal{F}_{\mathrm{pr}}
$$

giving the desired result.

The proof of (3.6) when $-J<K<-J / \rho$ is obtained by the symmetry $Z^{+} \rightarrow Z^{-}, Z^{-} \rightarrow Z^{+}$when $K \rightarrow-K$ and thus we take

$$
2 \nu \kappa \max \left(e^{-\beta\left(\frac{J-|K|}{2}\right)}, e^{-\beta\left(\frac{\rho|K|-J}{1+\rho}\right)}, 8 e(e-1) \kappa \nu^{2} e^{-\beta|K|}\right) \leq 1
$$

\section{Acknowledgments}

The authors thank Charles Pfister for useful discussions. C.D. and J.R. acknowledge the Centre de Recherche en Modélisation Moléculaire-Université de Mons-Hainaut for warm hospitality and financial support.

\section{References}

[1] D.B. Abraham and L.F. Ko, Exact derivation of the modified Young equation for partial wetting, Phys. Rev. Lett. 63, 275-279 (1989).

[2] C. Borgs, J. De Coninck, and R. Kotecký, An equilibrium lattice model of wetting on rough substrates, J. Stat. Phys. 94, 299-320 (1999).

[3] C. Borgs, J. De Coninck, R. Kotecký, and M. Zinque, Does the roughness of the substrate enhance wetting, Phys. Rev. Lett. 74, 2292-2294 (1995).

[4] J. Bricmont, J.L. Lebowitz and C.-E. Pfister, On the surface tension of lattice systems, Ann. N. Y. Acad. Soc. 337, 214 (1980).

[5] J. Bricmont, J.L. Lebowitz and C.-E. Pfister, Non-translation invariant Gibbs states with coexisting phases II, Commun. Math. Phys. 66, 21 (1979); Non-translation invariant Gibbs states with coexisting phases III, Commun. Math. Phys. 66, 21 (1979). 
[6] J. Bricmont, J.L. Lebowitz, C.-E. Pfister, and E. Olivieri, Nontranslation invariant Gibbs states with coexisting phases I, Commun. Math. Phys. 66, 1 (1979).

[7] A. B. D. Cassie, Discuss. Faraday Soc. 57, 5041 (1952).

[8] J. De Coninck and F. Dunlop, Partial to complete wetting: A microscopic derivation of the Young relation, J. Stat. Phys. 47, 827 (1987).

[9] J. De Coninck, F. Dunlop, and V. Rivasseau, On the microscopic validity of the Wulff construction and of the generalized Young equation, Commun. Math. Phys. 121, 401 (1989).

[10] J. De Coninck, S. Miracle-Solé, and J. Ruiz, The generalized Young's equation for rough and heterogeneous substrates : A microscopic proof, Phys. Rev. E 65, 0361XX (2002).

[11] J. De Coninck, S. Miracle-Solé, and J. Ruiz, Rigorous generalization of Young's law for heterogeneous and rough substrates, J. Stat. Phys. 111, 17 (2003).

[12] C. Dobrovolny, L. Laanait, and J. Ruiz, Surface transition of the semiinfinite Potts model I: The high bulk temperature regime, to appear in J. Stat. Phys..

[13] R. L. Dobrushin, Estimates of semi-invariants for the Ising model at low temperatures, Amer. Math. Soc. Transl. 177, 59-81 (1996).

[14] R. L. Dobrushin, Gibbs states describing the coexistence of phases for a three-dimensional Ising model, Theory Prob. Appl. 17, 582 (1972).

[15] F. Dunlop and K. Topolski, Cassie's law and concavity of wall tension with respect to disorder, J. Stat. Phys. 98, 1115-1134 (2000).

[16] J. Fröhlich and C.E. Pfister, Semi-infinite Ising model I: Thermodynamic functions and phase diagram in absence of magnetic field, Commun. Math. Phys. 109, 493 (1987); Semi-infinite Ising model II: The wetting and layering transition, Commun. Math. Phys. 112, 112 (1987); and The wetting and layering transitions in the half-infinite Ising model, Europhys. Lett. 3, 845 (1987). 
[17] G. Gallavotti, A. Martin Löf, and S. Miracle-Solé, Some problems connected with the coexistence of phases in the Ising model, in "Statistical mechanics and mathematical problems", Lecture Notes in Physics vol 20, pp. 162-204, Springer, Berlin (1973).

[18] P. Holicky, R. Kotecky, and M. Zahradnik, Rigid interfaces for lattice models at low temperatures, J. Stat. Phys. 50, 755 (2002).

[19] R. Kotecký and D. Preiss, Cluster Expansion for Abstract Polymer Models, Commun. Math. Phys. 103491 (1986).

[20] S. Miracle-Solé, On the convergence of cluster expansion, Physica A 279, 244 (2000).

[21] S. Miracle-Solé, Surface tension, Step free energy, and facets in the equilibrium crystal, J. Stat. Phys. 79, 183 (1995).

[22] S. Miracle-Solé and J. Ruiz, On the Wulff construction as a problem of equivalence of statistical ensembles, in "On Three levels", M. Fannes et al. eds., Plenum Press, New York (1994).

[23] C.-E. Pfister and O. Penrose, Commun. Math. Phys. Analyticity properties of the surface free energy of the Ising model, Commun. Math. Phys. 115, 691 (1988).

[24] C.-E. Pfister and Y. Velenik, Mathematical theory of the wetting phenomenon in the 2D Ising model, Helv. Phys. Acta 69, 949 (1996).

[25] P. Roura and J. Fort, Comments on "Effects of the surface roughness on sliding angles of water droplets on superhydrophobic surfaces", Langmuir 98, 566 (2002).

[26] P. S. Swain and R. Lipowski, Contact angles on heterogeneous surfaces: a new look at Cassie's and Wenzel's laws, Langmuir 14, 6772 (1998).

[27] K. Topolski, D. Urban, S. Brandon, and J. De Coninck, Influence of the geometry of a rough substrate on wetting, Phys. Rev. E 56, 3353 (1997).

[28] D. Urban, K. Topolski, and J. De Coninck, Wall tension and heterogeneous substrate, Phys. Rev. Lett. 76, 4388 (1996).

[29] R. N. Wenzel, Ind. Eng. Chem. 28, 988 (1936); J. Phys. 53, 1466 (1949). 


\section{Figure captions}

1. Young's contact angle

2. The substrate surface $\partial W$.

3. Dependence of the equilibrium contact angle $\theta$ on the "density of pores" $c^{\prime}$ exhibiting a transition between a Wenzel's regime (1) and a Cassie's regime (2).

4. The diagram of ground states. 


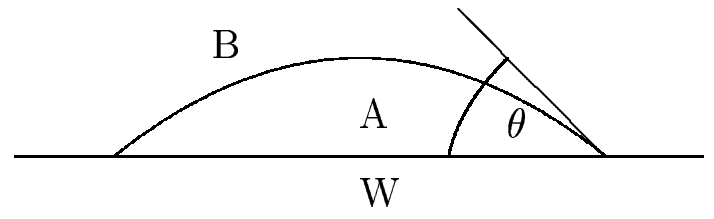




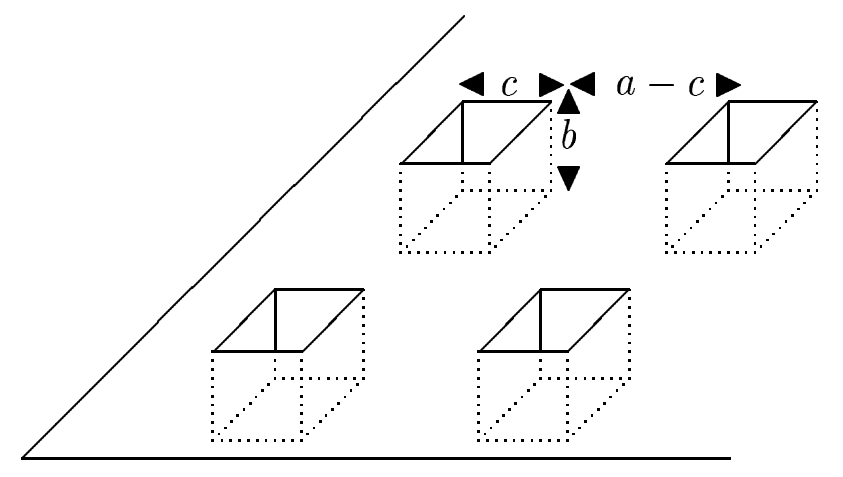




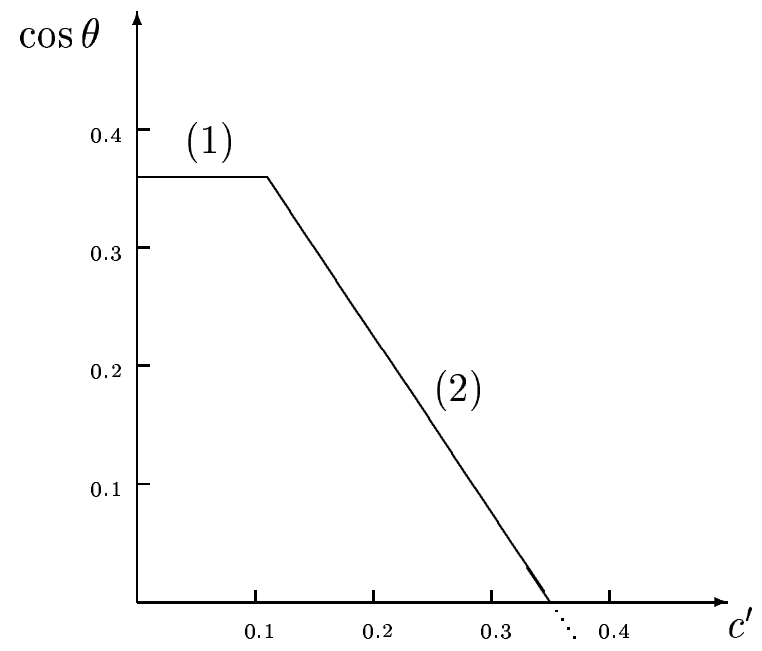




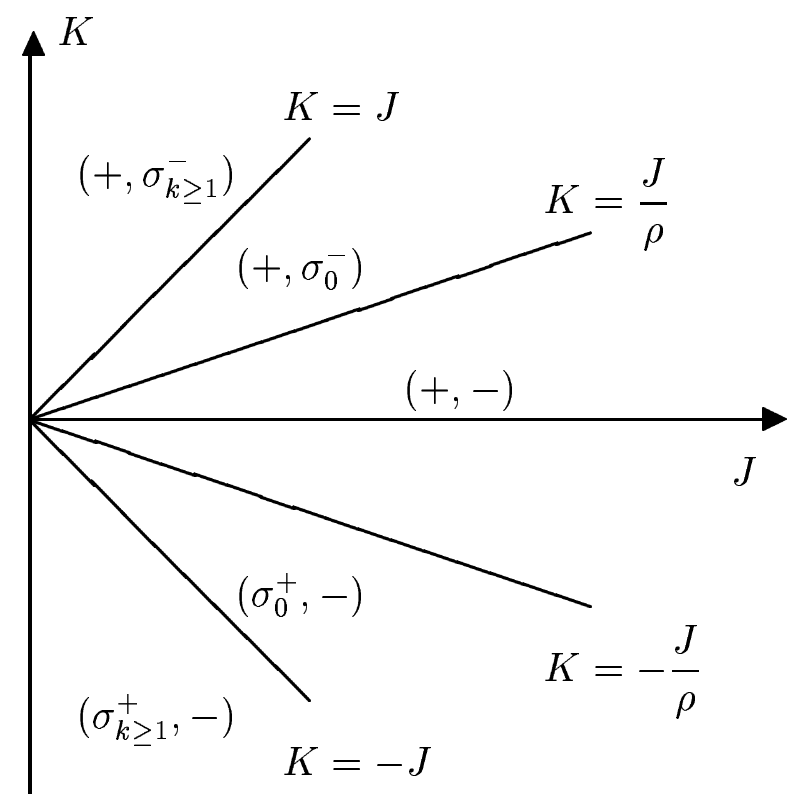

